\title{
AN ACTIVE-SET EQUALITY CONSTRAINED NEWTON SOLVER WITH FEASIBILITY RESTORATION FOR INVERSE COEFFICIENT PROBLEMS IN ELLIPTIC VARIATIONAL INEQUALITIES
}

\author{
M. HINTERMÜLLER*
}

\begin{abstract}
An output least-squares formulation for a class of parameter identification problems for elliptic variational inequalities is considered. Based on the concept of C-stationarity an active set type solver with feasibility restoration is introduced. It is shown that the new method relates to so-called implicit programming techniques in the context of mathematical programs with equilibrium constraints. In the discrete setting, in order to overcome the ill-posedness of the problem, the parameter of interest is discretized on a coarser mesh than the state of the system. In addition, if the parameter corresponds to the coefficient in the bilinear form of the underlying differential operator, an interior point treatment is employed to maintain the coercivity of the elliptic operator. Moreover, the computational domain for the coefficient depends on the measurement data. The paper ends by a report on numerical tests including an application to a simplified lubrication problem in a rolling element device.
\end{abstract}

Key words. Active set algorithm, bilevel optimization, optimal control of variational inequalities, parameter identification, variational inequality

AMS subject classifications. 34A55, 47J06, 49J24, 90C26

1. Introduction. Many practical applications ranging from engineering science to mathematical finance are modeled properly by variational inequalities (VIs). Particular instances comprise contact problems in elasticity [15] or problems in elastoplacticity [9], elastohydrodynamic lubrication problems [6, 8, 14], or Black-Scholes models for American options [1, 2]. Based on measurements of the state of the underlying system, frequently one is interested in obtaining estimates of some parameter involved in the variational inequality model. In elasticity this might be estimates of material properties based on measurements of displacements, in elastohydrodynamic lubrication one might be interested in estimating the gap height between two rotating surfaces from measurements of the pressure of the lubricant separating the surfaces, or in mathematical finance the reconstruction of the volatility of an underlying asset based on market data could be desirable. In all of these cases, one has to deal with a parameter estimation problem for a variational inequality with the parameter of interest occurring in the leading part of the differential operator.

In practice, parameter estimation problems are often treated by means of output least-squares formulations. In our context, in an abstract setting this results in the following minimization problem:

$$
\begin{array}{ll}
\operatorname{minimize} & F(y)+\alpha R(u) \quad \text { over }(y, u) \in \mathcal{Y} \times \mathcal{U} \\
\text { subject to } & u \in U_{\mathrm{ad}}, \\
& y \in \operatorname{SVI}(u)
\end{array}
$$

where $\mathcal{Y}$ and $\mathcal{U}$ denote the Hilbert spaces of the state $y$ and the parameter $u$, and $U_{\text {ad }} \subset \mathcal{U}$ represents a feasible parameter range. Further, $F$ is supposed to be bounded from below and sufficiently smooth, and $R$ usually corresponds to some regularization (with regularization parameter $\alpha>0$ )

\footnotetext{
${ }^{*}$ University of Sussex, Department of Mathematics, Falmer, Brighton, United Kingdom (m.hintermueller@sussex.ac.uk). The author acknowledges support by the Austrian Ministry for Science and Education and the Austrian Science Fund FWF under START-program Y013 "Interfaces and Free Boundaries".
} 
and it is assumed to be lower semicontinuous with

$$
R(u) \geq c_{1}\|u\|_{\mathcal{U}}+c_{2} \quad \forall u \in \mathcal{U}
$$

for some constants $c_{1}, c_{2} \in \mathbb{R}$ with $c_{1} \geq 0$. By $\operatorname{SVI}(u)$ we denote the solution set of the underlying variational inequality which depends on the parameter $u$. Throughout we assume that, given $u$, the VI admits a unique solution $y(u) \in \mathcal{Y}$, i.e., $\operatorname{SVI}(u)$ is single-valued.

In contrast to parameter identification problems in partial differential equations (PDEs), problems of the type (1.1) are significantly less researched. The theoretical as well as the numerical treatment of (1.1) have to cope with several difficulties: (i) Identifyability results or (regularization) parameter selection rules depending on the noise level in the given data are complicated by the presence of constraints on $y$. In fact, as an example consider the elliptic VI problem

$$
\text { Find } y \in K \text { such that }\langle A(u) y-f, v-y\rangle \geq 0 \quad \forall v \in K \text {, }
$$

where $K=\left\{y \in H_{0}^{1}(\Omega): y \geq 0\right.$ a.e. in $\left.\Omega\right\} \subset \mathcal{Y}=: H_{0}^{1}(\Omega)$ is closed and convex,

$$
A(u)=\operatorname{div}(c(u) \nabla y)
$$

denotes a second order elliptic differential operator depending on $u$ with $c: \mathbb{R} \rightarrow \mathbb{R}_{++}$sufficiently smooth and $f \in L^{2}(\Omega)$ for a bounded domain $\Omega \subset \mathbb{R}^{n}$ with sufficiently smooth boundary $\partial \Omega$. The solution $y=y(u)$ of $(1.2)$ induces the active or coincidence set

$$
\mathcal{A}=\{x \in \Omega: y(x)=0\}
$$

Considering the structure of $A$, we immediately find that an identification of $u$ on $\mathcal{A}$ is impossible, in general. Indeed, while for PDEs (instead of VIs in (1.1)) the singular set $\{x \in \Omega: \nabla y(x)=0\}$ is often of measure zero, this is usually not the case for VIs of the type (1.2). (ii) From an optimization theoretic point of view (1.1) belongs to the problem class of mathematical programs with equilibrium constraints (MPECs; more precisely, it is a mathematical program with complementarity constraints); see [18] and the many references therein. It is known that this problem class is difficult to handle for several reasons. First, the existence of Lagrangian multipliers for characterizing optimality does not follow from standard theory due to the lack of constraint qualifications. Rather one has to find alternative approaches to guarantee multipliers. As a result, one has to handle several distinct stationarity concepts such as weak, B-, C-, M-, or strong stationarity [24, 25]. Secondly, the structure of the feasible set might be of combinatorial nature, which complicates the numerical treatment significantly. Thirdly, the development of solution algorithms is delicate due to the aforementioned difficulties and the large scale character of the discrete problem in our context.

In this paper we primarily focus on the last aspect mentioned above. In fact, we introduce a solution algorithm for (1.1) which improves earlier methods like the one in [10]. Based on the Cstationarity concept, the new method aims at avoiding numerical difficulties due to multipliers with low regularity by employing an active set approach. It uses a feasibility restoration for stabilization and a Newton-type linearization of the remaining equations in the first order characterization of solutions to (1.1) for obtaining update directions. Our analysis shows that this method is closely related to the implicit programming technique $[19,20,21]$, which is a nonsmooth minimization approach to $(1.1)$. In the present context the nonsmoothness arises due to the fact that $y(\cdot)$ need not be Fréchet differentiable.

The remaining sections are organized as follows: In the next section we specify our model problem and discuss a suitable stationarity concept which will then be the basis for the algorithmic 
development. Our active set type algorithm is introduced in section 3. Further it is related to the implicit programming approach in MPECs and its convergence is discussed. Finally, in section 4 we report on numerical results. The implementation of our algorithm relies on a nested iteration environment in order to speed up convergence.

2. C-stationarity for output-least-squares formulation. All of our subsequent considerations are based on the following version of the output-least-squares problem (1.1):

$$
\operatorname{minimize} \quad J(y, u):=\frac{1}{2}\left\|y-y_{m}\right\|_{L^{2}(\Omega)}^{2}+\frac{\alpha}{2}\|u\|_{H^{1}(\Omega)}^{2} \text { over }(y, u) \in \mathcal{Y}:=H_{0}^{1}(\Omega) \times \mathcal{U}:=H^{1}(\Omega)
$$

subject to $u \in U_{\text {ad }}:=\left\{u \in H^{1}(\Omega): \epsilon_{l} \leq u \leq \epsilon_{u}\right.$ a.e. in $\left.\Omega\right\}$,

$$
y \in K, \quad a(y, v-y ; u)-(f(u), v-y) \geq 0 \forall v \in K
$$

with measurement data $y_{m} \in L^{2}(\Omega)$, and $\alpha, \epsilon_{l}, \epsilon_{u} \in \mathbb{R}_{++}, \epsilon_{l}<\epsilon_{u}$, as well as the cone

$$
K:=\left\{v \in H_{0}^{1}(\Omega): v \geq 0 \text { a.e. in } \Omega\right\}
$$

and the bilinear form $a$ and source term $f$ given by

$$
a(y, w ; u)=\int_{\Omega} c(u) \nabla y \cdot \nabla w d x, \quad f(u)=B u+g,
$$

where $c: \mathbb{R}_{++} \rightarrow \mathbb{R}_{++}$is of class $C^{2}, c(u) \geq \epsilon_{c}>0$ a.e. in $\Omega$ for $u \in U_{\text {ad }}, B \in L\left(\mathcal{U}, L^{2}(\Omega)\right)$, and $g \in L^{2}(\Omega)$. Further, $\Omega \subset \mathbb{R}^{n}, n \leq 2$, is a bounded domain with sufficiently regular boundary $\Gamma:=\partial \Omega$. By $(\cdot, \cdot)$ we denote the usual $L^{2}$-inner product. Referring back to $(1.1)$ we see that $F(y)=\frac{1}{2}\left\|y-y_{m}\right\|_{L^{2}(\Omega)}^{2}$ and $R(u)=\frac{1}{2}\|u\|_{H^{1}(\Omega)}^{2}$. Due to the resemblance of (1.1) to optimal control problems for variational inequalities (in our case with the control also acting in the coefficient of the bilinear form) [3], we sometimes refer to $y$ as the state and to $u$ as the control variable, respectively.

It is well-known that given $u$ the VI in (P) admits a unique solution $y(u) \in K$; see, e.g., [16]. We also note that the VI can be reformulated by introducing a slack variable (Lagrange multiplier) $\lambda$ resulting in

$$
\begin{aligned}
& a(y, v ; u)-(f(u), v)-\langle\lambda, v\rangle=0 \quad \forall v \in H_{0}^{1}(\Omega) \\
& \langle\lambda, v\rangle \geq 0 \quad \forall v \in K, \quad y \in K, \quad\langle\lambda, y\rangle=0
\end{aligned}
$$

where $\langle\cdot, \cdot\rangle$ denotes the duality paring between $H_{0}^{1}(\Omega)$ and its dual $H^{-1}(\Omega)$.

For the subsequent development we further recall that due to [23, Chap. 5, Thm. 2.5] (see also [5, Chap. 1, Thm. 4.2] for a related context) we have

$$
\|y(u)\|_{W_{0}^{1, r}} \leq \beta\|f(u)\|_{W^{-1, r}(\Omega)}
$$

for some $r>2$ and some constant $\beta>0$. Here and below $\beta$ denotes a generic positive constant which can take different values on different occasions.

Now, the existence of a solution $\left(y^{*}, u^{*}\right) \in K \times U_{\text {ad }}$ of $(\mathrm{P})$ follows from standard arguments. In fact, $U_{\text {ad }}$ is weakly closed such that every minimizing sequence $\left\{u_{k}\right\}$ in $U_{\text {ad }}$ admits a weak accumulation point $u^{*}$ in $U_{\text {ad }}$. Without loss of generality we assume that the corresponding subsequence is again denoted by $\left\{u_{k}\right\}$. From the objective functional in (P) and the estimate (2.3) we obtain the uniform boundedness of $\left\{y\left(u_{k}\right)\right\}$ in $H_{0}^{1}(\Omega)$. Then Theorem A.1 yields that $y\left(u_{k}\right)$ converges to $y\left(u^{*}\right)$ 
strongly in $H_{0}^{1}(\Omega)$. For this to hold true, again the estimate (2.3) is of importance. The strong convergence of $\left\{y\left(u_{k}\right)\right\}$ together with the weak lower semicontinuity of the objective functional in (P) guarantee the existence of $\left(y^{*}, u^{*}\right)$.

In the sequel we utilize the proof technique of [10] for obtaining C-stationarity conditions for (P); see Theorem 2.1 below. The first step consists in replacing the VI by a regularized version. For this purpose we first note that the VI represents the first order optimality system of

$$
\text { minimize } J_{\ell}(y ; u)=\frac{1}{2} a(y, y ; u)-(f(u), y) \quad \text { over } \quad y \in K \text {. }
$$

Here the subscript $\ell$ refers to the fact that this problem is a so-called lower level optimization problem as it occurs as a constraint in the overall minimization problem $(\mathrm{P})$. Note that $u$ enters (2.4) as a parameter. By transforming the hard constraint $y \geq 0$ into a soft constraint, the following regularized lower level problem is obtained:

$$
\operatorname{minimize} J_{\ell}^{\gamma}(y ; u)=\frac{1}{2} a(y, y ; u)-(f(u), y)+\frac{1}{2 \gamma}\|\max (0, \bar{\lambda}-\gamma y)\|_{L^{2}(\Omega)}^{2} \quad \text { over } \quad y \in H_{0}^{1}(\Omega),
$$

where $\bar{\lambda} \geq \max (0,-f(u))$ for all $u \in U_{\text {ad }}, \bar{\lambda} \in L^{2}(\Omega)$, denotes a fixed shift parameter. Due to the uniform convexity of $J_{\ell}^{\gamma}$ there exists a unique solution $y_{\gamma}(u) \in H_{0}^{1}(\Omega)$ of (2.5). Note the difference between (2.5) and (2.4): In (2.5) the constraint $y \geq 0$ is no longer explicit rather its (shifted) violation in $L^{2}(\Omega)$ is penalized by $\gamma>0$. The first order necessary and (due to convexity) sufficient condition is

$$
a(y, v ; u)-(f(u), v)-(\max (0, \bar{\lambda}-\gamma y), v)=0 \quad \forall v \in H_{0}^{1}(\Omega) .
$$

In view of $(2.1)$ we find that $\max \left(0, \bar{\lambda}-\gamma y_{\gamma}(u)\right) \in L^{2}(\Omega)$ represents a regular approximation of the Lagrange multiplier $\lambda(u)$ at $y(u)$. In what follows, to ease the notation, we use $(w)^{+}$instead of $\max (0, w)$. Choosing $v:=\left(-y_{\gamma}(u)\right)^{+} \in H_{0}^{1}(\Omega)$ in $(2.6)$ one finds

$$
0 \leq-\epsilon_{c}\left\|\left(-y_{\gamma}(u)\right)^{+}\right\|_{H_{0}^{1}(\Omega)}^{2}-\left(f(u)+(-f(u))^{+},\left(-y_{\gamma}(u)\right)^{+}\right)-\gamma\left\|\left(-y_{\gamma}(u)\right)^{+}\right\|_{L^{2}(\Omega)}^{2} \leq 0,
$$

which implies $y_{\gamma}(u) \geq 0$. Thus, the $\bar{\lambda}$-shift yields feasibility of $y_{\gamma}(u)$. The regularized version of (P) now reads

$$
\begin{array}{cl}
\operatorname{minimize} & \frac{1}{2}\left\|y-y_{m}\right\|_{L^{2}(\Omega)}^{2}+\frac{\alpha}{2}\|u\|_{H^{1}(\Omega)}^{2} \quad \text { over }(y, u) \in H_{0}^{1}(\Omega) \times H^{1}(\Omega) \\
\text { subject to } & u \in U_{\mathrm{ad}}:=\left\{u \in H^{1}(\Omega): \epsilon_{l} \leq u \leq \epsilon_{u} \text { a.e. in } \Omega\right\}, \\
& a(y, v ; u)-(f(u), v)-\left((\bar{\lambda}-\gamma y)^{+}, v\right)=0 \quad \forall v \in H_{0}^{1}(\Omega)
\end{array}
$$

for some $\gamma>0$. Before we commence with studying $\left(\mathrm{P}_{\gamma}\right)$, which is the second step in the proof of stationarity, we focus on the regularized lower level problem (2.5) and its consistency. We have

$$
\frac{1}{2 \gamma}\|\bar{\lambda}\|_{L^{2}(\Omega)}^{2}=J_{\ell}^{\gamma}(0 ; u) \geq J_{\ell}^{\gamma}\left(y_{\gamma}(u) ; u\right) \geq J_{\ell}\left(y_{\gamma}(u) ; u\right) \geq J_{\ell}(y(u) ; u),
$$

and further, by feasibility of $y_{\gamma}(u),\left\|\left(\gamma^{-1} \bar{\lambda}-y_{\gamma}(u)\right)^{+}\right\|_{L^{2}(\Omega)} \rightarrow 0$ as $\gamma \rightarrow \infty$. Hence, the penalization is consistent with the original lower level problem. Due to the monotonicity of the max-term in (2.6), similar to (2.3) we have

$$
\left\|y_{\gamma}(u)\right\|_{W_{0}^{1, r}(\Omega)} \leq \beta\left(\|f(u)\|_{W^{-1, r}(\Omega)}+\|\bar{\lambda}\|_{L^{2}(\Omega)}\right)
$$


for some $r>2$ and some constant $\beta>0$, which is independent of $\gamma$. The analogue of Theorem A.1 in the appendix yields $y_{\gamma}\left(u_{k}\right) \rightarrow y_{\gamma}(u)$ strongly in $H_{0}^{1}(\Omega)$ (and uniformly in $\gamma$ ) for $u_{k} \rightarrow u$ weakly in $H^{1}(\Omega)$. Lemma 3.1 in [10] then guarantees the strong $H_{0}^{1}(\Omega)$-convergence of $\left\{y_{\gamma}(u)\right\}$ to $y(u)$, the unique solution of (2.4). Now we are ready to turn to $\left(\mathrm{P}_{\gamma}\right)$. In fact, with this preparatory work on the regularized lower level problem, similar to $(\mathrm{P})$ one argues the existence of a solution $\left(y_{\gamma}^{*}, u_{\gamma}^{*}\right) \in H_{0}^{1}(\Omega) \times H^{1}(\Omega)$. It remains to prove consistency of $\left(\mathrm{P}_{\gamma}\right)$. For this purpose note that

$$
\frac{1}{2}\left\|y_{\gamma}^{*}-y_{m}\right\|_{L^{2}(\Omega)}^{2}+\frac{\alpha}{2}\left\|u_{\gamma}^{*}\right\|_{H^{1}(\Omega)}^{2} \leq \frac{1}{2}\left\|y_{\gamma}(u)-y_{m}\right\|_{L^{2}(\Omega)}^{2}+\frac{\alpha}{2}\|u\|_{H^{1}(\Omega)}^{2}
$$

for arbitrarily fixed $u \in U_{\text {ad }}$. From this we immediately conclude the uniform boundedness of $\left\{u_{\gamma}^{*}\right\}$ in $H^{1}(\Omega)$ and the existence of a weakly convergent subsequence (the weak limit point is denoted by $\left.u^{*}\right)$. Without loss of generality we denote this subsequence again by $\left\{u_{\gamma}^{*}\right\}$. Further,

$$
\left\|y_{\gamma}^{*}-y^{*}\right\|_{H_{0}^{1}(\Omega)} \leq\left\|y_{\gamma}^{*}-y_{\gamma}\left(u^{*}\right)\right\|_{H_{0}^{1}(\Omega)}+\left\|y_{\gamma}\left(u^{*}\right)-y^{*}\right\|_{H_{0}^{1}(\Omega)} \rightarrow 0 \text { for } \gamma \rightarrow \infty .
$$

Once the strong $H_{0}^{1}(\Omega)$-convergence of $y_{\gamma}^{*}$ is established one readily finds that the VI is satisfied in the limit. Then (2.8) yields optimality.

Although we have already employed a lower level regularization, the problem $\left(\mathrm{P}_{\gamma}\right)$ is still difficult to handle. This is due to the non-differentiable equality constraint, i.e., the first order optimality condition of the regularized lower level problem, which continues to cause problems with respect to the existence of Lagrange multipliers. In order to overcome this difficulty, as the third proof step for obtaining stationarity conditions the following smooth approximation is introduced:

$\left(\mathrm{P}_{\gamma, \epsilon}\right)$

$$
\text { minimize } \frac{1}{2}\left\|y-y_{m}\right\|_{L^{2}(\Omega)}^{2}+\frac{\alpha}{2}\|u\|_{H^{1}(\Omega)}^{2} \quad \text { over }(y, u) \in H_{0}^{1}(\Omega) \times H^{1}(\Omega)
$$

subject to $u \in U_{\mathrm{ad}}:=\left\{u \in H^{1}(\Omega): \epsilon_{l} \leq u \leq \epsilon_{u}\right.$ a.e. in $\left.\Omega\right\}$,

$$
a(y, v ; u)-(f(u), v)-\left(\max _{\epsilon}(0, \bar{\lambda}-\gamma y), v\right)=0 \quad \forall v \in H_{0}^{1}(\Omega),
$$

where $\max _{\epsilon}(0, \cdot)$ is a $C^{1}$-regularized version of the max-function. We use

$$
\max _{\epsilon}(0, s)= \begin{cases}s & \text { for } s>\frac{\epsilon}{2}, \\ \frac{1}{2 \epsilon}\left(s+\frac{\epsilon}{2}\right)^{2} & \text { for }|s| \leq \frac{\epsilon}{2}, \\ 0 & \text { for } s<-\frac{\epsilon}{2}\end{cases}
$$

It follows from standard arguments that $\left(\mathrm{P}_{\gamma, \epsilon}\right)$ admits a solution $\left(y_{\gamma, \epsilon}^{*}, u_{\gamma, \epsilon}^{*}\right)$. The choice of $\bar{\lambda}$ yields $y_{\gamma, \epsilon}^{*} \geq 0$ a.e. in $\Omega$. Following, e.g., [27] one readily finds the first order optimality characterization

$$
\begin{aligned}
\left(y_{\gamma, \epsilon}^{*}-y_{m}, v\right)+a\left(p_{\gamma, \epsilon}^{*}, v ; u_{\gamma, \epsilon}^{*}\right)+\left(\mu_{\gamma, \epsilon}^{*}, v\right)=0 & \forall v \in H_{0}^{1}(\Omega), \\
\left\langle\alpha u_{\gamma, \epsilon}^{*}+a_{u}\left(y_{\gamma, \epsilon}^{*}, p_{\gamma, \epsilon}^{*} ; u_{\gamma, \epsilon}^{*}\right)-B^{\star} p_{\gamma, \epsilon}^{*}, w-u_{\gamma, \epsilon}^{*}\right\rangle_{H^{1}(\Omega)^{\prime}, H^{1}(\Omega)} \geq 0 & \forall w \in U_{\mathrm{ad}}, \\
a\left(y_{\gamma, \epsilon}^{*}, v ; u_{\gamma, \epsilon}^{*}\right)-\left(\lambda_{\gamma, \epsilon}^{*}, v\right)-\left(f\left(u_{\gamma, \epsilon}^{*}\right), v\right) & =0 \quad \forall v \in H_{0}^{1}(\Omega),
\end{aligned}
$$

with the adjoint state $p_{\gamma, \epsilon}^{*} \in H_{0}^{1}(\Omega)$. Above, we use

$$
\begin{aligned}
\mu_{\gamma, \epsilon}^{*} & :=\gamma \max _{\epsilon}^{\prime}\left(0, \bar{\lambda}-\gamma y_{\gamma, \epsilon}^{*}\right) p_{\gamma, \epsilon}^{*}, \\
\lambda_{\gamma, \epsilon}^{*} & :=\max _{\epsilon}\left(0, \bar{\lambda}-\gamma y_{\gamma, \epsilon}^{*}\right),
\end{aligned}
$$

and $a_{u} \in\left(H^{1}(\Omega)\right)^{\prime}$ denotes the derivative of $a$ with respect to $u$. 
Finally, it remains to study the convergence as $\gamma \rightarrow \infty$ and $\epsilon \rightarrow 0$ for appropriately linked $\epsilon$ and $\gamma$. First note that picking $\hat{u} \equiv \epsilon_{u}$ we obtain $\hat{y}_{\gamma, \epsilon}=y_{\gamma, \epsilon}(\hat{u})$ from the equality constraint in $\left(\mathrm{P}_{\gamma, \epsilon}\right)$, and further $\left\|\hat{y}_{\gamma, \epsilon}\right\|_{H_{0}^{1}(\Omega)} \leq \beta$ with $\beta$ independent of $\gamma$ and $\epsilon$. Next, for $J(y, u)=\frac{1}{2}\left\|y-y_{m}\right\|_{L^{2}(\Omega)}^{2}+$ $\frac{\alpha}{2}\|u\|_{H^{1}(\Omega)}^{2}$ we have

$$
J\left(y_{\gamma, \epsilon}^{*}, u_{\gamma, \epsilon}^{*}\right) \leq J\left(\hat{y}_{\gamma, \epsilon}, \hat{u}\right) \leq \beta .
$$

for all $\gamma$ and $\epsilon$. Hence, $\left\|u_{\gamma, \epsilon}^{*}\right\|_{H^{1}(\Omega)}$ is uniformly bounded. Thus, there exists a weakly convergent subsequence, which we again denote by $\left\{u_{\gamma, \epsilon}^{*}\right\}$ without loss of generality. Since $y_{\gamma, \epsilon}^{*}=y_{\gamma, \epsilon}\left(u_{\gamma, \epsilon}^{*}\right)$, we have that $\left\{y_{\gamma, \epsilon}^{*}\right\}$ converges strongly in $H_{0}^{1}(\Omega)$. Now, similar arguments as for $\left(\mathrm{P}_{\gamma}\right)$ yield $\left(y_{\gamma, \epsilon}^{*}, u_{\gamma, \epsilon}^{*}\right) \rightarrow$ $\left(y^{*}, u^{*}\right)$ strongly-weakly in $H_{0}^{1}(\Omega) \times H^{1}(\Omega)$. Then (2.11) implies that $\lambda_{\gamma, \epsilon}^{*}$ is uniformly bounded in $H^{-1}(\Omega)$. A weak accumulation point $\lambda^{*}$ satisfies

$$
a\left(y^{*}, v ; u^{*}\right)-\left\langle\lambda^{*}, v\right\rangle-\left(f\left(u^{*}\right), v\right)=0 \quad \forall v \in H_{0}^{1}(\Omega) .
$$

Moreover, since $\lambda_{\gamma, \epsilon}^{*} \geq 0$ for all $\gamma$ and $\epsilon$, we have

$$
\left\langle\lambda^{*}, v\right\rangle \geq 0 \quad \forall v \in K \text {. }
$$

We also have

$$
\left\langle\lambda^{*}, y^{*}\right\rangle=0
$$

Indeed, we obtain the estimate

$$
\left|\left\langle\lambda_{\gamma, \epsilon}^{*}, y_{\gamma, \epsilon}^{*}\right\rangle\right| \leq\left|\int_{S_{1}} \frac{1}{2 \epsilon}\left(\bar{\lambda}-\gamma y_{\gamma, \epsilon}^{*}+\frac{\epsilon}{2}\right)^{2} y_{\gamma, \epsilon}^{*} d x\right|+\left|\int_{S_{2}}\left(\bar{\lambda}-\gamma y_{\gamma, \epsilon}^{*}\right) y_{\gamma, \epsilon}^{*} d x\right|
$$

where

Note that

$$
S_{1}:=\left\{\left|\bar{\lambda}-\gamma y_{\gamma, \epsilon}^{*}\right| \leq \frac{\epsilon}{2}\right\} \quad \text { and } \quad S_{2}=\left\{\bar{\lambda}-\gamma y_{\gamma, \epsilon}^{*}>\frac{\epsilon}{2}\right\}
$$

Hence, the right hand side in $(2.15)$ is of the order $O(1 / \gamma)$ which, together with the strong $H_{0}^{1}$ convergence of $y_{\gamma, \epsilon}^{*}$, yields $(2.14)$.

Choosing $v=p_{\gamma, \epsilon}^{*}$ in $(2.9)$ and considering $\max _{\epsilon}^{\prime}(0, w) \geq 0$ for all $w \in L^{2}(\Omega)$, we obtain the uniform (in $\gamma$ and $\epsilon$ ) boundedness of $\left\{p_{\gamma, \epsilon}^{*}\right\}$ in $H_{0}^{1}(\Omega)$ and further

$$
0 \leq \epsilon_{c}\left\|p_{\gamma, \epsilon}^{*}\right\|_{H_{0}^{1}(\Omega)}^{2}+\left(\mu_{\gamma, \epsilon}^{*}, p_{\gamma, \epsilon}^{*}\right) \leq\left(y_{\gamma, \epsilon}^{*}-y_{m}, p_{\gamma, \epsilon}^{*}\right) \leq \beta,
$$

where $\beta>0$ is independent of $\gamma$. This fact is important for the following considerations: Using $0 \leq \max _{\epsilon}^{\prime}\left(0, \bar{\lambda}-\gamma y_{\gamma, \epsilon}^{*}\right)(x) \leq 1$ for almost all $x \in S_{1}$ as well as (2.16) and (2.17), we obtain

$$
\begin{aligned}
\mid \int_{S_{1}} \gamma \max _{\epsilon}^{\prime}(0, \bar{\lambda} & \left.-\gamma y_{\gamma, \epsilon}^{*}\right) p_{\gamma, \epsilon}^{*} y_{\gamma, \epsilon}^{*} d x \mid \leq \gamma\left\|\max _{\epsilon}^{\prime}\left(0, \bar{\lambda}-\gamma y_{\gamma, \epsilon}^{*}\right) p_{\gamma, \epsilon}^{*}\right\|_{L^{2}\left(S_{1}\right)}\left\|y_{\gamma, \epsilon}^{*}\right\|_{L^{2}\left(S_{1}\right)} \\
& \leq \gamma^{-1 / 2}\left(\int_{S_{1}} \mu_{\gamma, \epsilon}^{*} p_{\gamma, \epsilon}^{*} d x\right)^{1 / 2}\|\bar{\lambda}+\epsilon / 2\|_{L^{2}\left(S_{1}\right)} \\
& \leq \gamma^{-1 / 2}\left\|y_{\gamma, \epsilon}^{*}-y_{m}\right\|_{L^{2}(\Omega)}^{1 / 2}\left\|p_{\gamma, \epsilon}^{*}\right\|_{L^{2}(\Omega)}^{1 / 2}\|\bar{\lambda}+\epsilon / 2\|_{L^{2}\left(S_{1}\right)} \\
& \leq \frac{\beta}{\sqrt{\gamma}}
\end{aligned}
$$


The adjoint equation (2.9) implies

$$
\gamma\left\|p_{\gamma, \epsilon}^{*}\right\|_{L^{2}\left(S_{2}\right)}^{2}+\left(\mu_{\gamma, \epsilon}^{*}, p_{\gamma, \epsilon}^{*}\right)_{L^{2}\left(\Omega \backslash S_{2}\right)}+\epsilon_{c}\left\|\nabla y_{\gamma, \epsilon}^{*}\right\|_{L^{2}(\Omega)}^{2} \leq\left\|y_{\gamma, \epsilon}^{*}-y_{m}\right\|_{L^{2}(\Omega)}\left\|p_{\gamma, \epsilon}^{*}\right\|_{L^{2}(\Omega)}
$$

which yields

$$
\left|\int_{S_{2}} \gamma \max _{\epsilon}^{\prime}\left(0, \bar{\lambda}-\gamma y_{\gamma, \epsilon}^{*}\right) p_{\gamma, \epsilon}^{*} y_{\gamma, \epsilon}^{*} d x\right| \leq\left\|p_{\gamma, \epsilon}\right\|_{L^{2}\left(S_{2}\right)}\|\bar{\lambda}+\epsilon / 2\|_{L^{2}\left(S_{2}\right)} \leq \frac{\beta}{\sqrt{\gamma}} .
$$

Combining (2.18) and (2.19) and recalling that $\max _{\epsilon}^{\prime}\left(0, \bar{\lambda}-\gamma y_{\gamma, \epsilon}^{*}\right)(x)=0$ for almost all $x \in \Omega \backslash$ $\left(S_{1} \cup S_{2}\right)$ we infer

$$
\lim _{\substack{\gamma \rightarrow \infty \\ \epsilon \rightarrow 0}}\left|\left\langle y_{\gamma, \epsilon}^{*}, \mu_{\gamma, \epsilon}^{*}\right\rangle\right|=0
$$

The uniform $H_{0}^{1}$-boundedness of $\left\{y_{\gamma, \epsilon}^{*}\right\}$ and $\left\{p_{\gamma, \epsilon}^{*}\right\}$, respectively, as well as $u_{\gamma, \epsilon}^{*} \in U_{\text {ad }} \subset L^{\infty}(\Omega)$ for all $\gamma, \epsilon$ yield

$$
\sup _{v \in H_{0}^{1}(\Omega),\|v\|_{H_{0}^{1}(\Omega)}=1}\left|\left\langle\mu_{\gamma, \epsilon}^{*}, v\right\rangle\right| \leq\left\|y_{\gamma, \epsilon}^{*}-y_{m}\right\|_{L^{2}(\Omega)}+\left\|c\left(u_{\gamma, \epsilon}^{*}\right)\right\|_{L^{\infty}(\Omega)}\left\|\nabla p_{\gamma, \epsilon}^{*}\right\|_{L^{2}(\Omega)} \leq \beta
$$

and, hence, the uniform boundedness of $\left\{\mu_{\gamma, \epsilon}^{*}\right\}$ in $H^{-1}(\Omega)$. From this fact and the strong $H_{0}^{1}$ convergence of $y_{\gamma, \epsilon}^{*}$ we obtain

$$
\lim _{\substack{\gamma \rightarrow \infty \\ \epsilon \rightarrow 0}}\left\langle y_{\gamma, \epsilon}^{*}, \mu_{\gamma, \epsilon}^{*}\right\rangle=\left\langle y^{*}, \mu^{*}\right\rangle=0
$$

where $\mu^{*} \in H^{-1}(\Omega)$ denotes the weak limit of $\left\{\mu_{\gamma, \epsilon}^{*}\right\}$.

Next we establish the complementarity relation

$$
\lim _{\substack{\gamma \rightarrow \infty \\ \epsilon \rightarrow 0}}\left\langle p_{\gamma, \epsilon}^{*}, \lambda_{\gamma, \epsilon}^{*}\right\rangle=0
$$

From the definition of $\lambda_{\gamma, \epsilon}^{*}$ we infer

$$
\int_{S_{1}}\left|p_{\gamma, \epsilon}^{*} \lambda_{\gamma, \epsilon}^{*}\right| d x=\int_{S_{1}}\left|p_{\gamma, \epsilon}^{*}\right| \frac{1}{2 \epsilon}\left(\bar{\lambda}-\gamma y_{\gamma, \epsilon}^{*}+\frac{\epsilon}{2}\right)^{2} d x \leq \frac{\epsilon}{2}\left\|p_{\gamma, \epsilon}^{*}\right\|_{L^{1}\left(S_{1}\right)} \leq \beta \epsilon \rightarrow 0 \quad \text { for } \epsilon \rightarrow 0 .
$$

The regularized adjoint equation (2.9) with $v=p_{\gamma, \epsilon}^{*}$ yields

$$
\left\|p_{\gamma, \epsilon}^{*}\right\|_{L^{2}\left(S_{2}\right)} \leq \frac{\beta}{\sqrt{\gamma}}
$$

Hence, we obtain

$$
\int_{S_{2}}\left|p_{\gamma, \epsilon}^{*} \lambda_{\gamma, \epsilon}^{*}\right| d x \leq\left\|p_{\gamma, \epsilon}^{*}\right\|_{L^{2}\left(S_{2}\right)}\left\|\bar{\lambda}-\gamma y_{\gamma, \epsilon}^{*}\right\|_{L^{2}\left(S_{2}\right)} \leq \beta\left\|p_{\gamma, \epsilon}^{*}\right\|_{L^{2}\left(S_{2}\right)} \rightarrow 0 \quad \text { for } \gamma \rightarrow \infty, \epsilon \rightarrow 0 .
$$

The limit (2.21) now follows from (2.22) and (2.23). 
Finally note that due to the definition of $\mu_{\gamma, \epsilon}^{*}$ and (2.9) we have

$$
0 \leq\left(\mu_{\gamma, \epsilon}^{*}, p_{\gamma, \epsilon}^{*}\right) \leq \beta
$$

for all $\gamma>0$ and $\epsilon>0$.

Before stating the main stationarity theorem we mention that the control-to-adjoint-state mapping $p_{\gamma, \epsilon}(\cdot)$ resulting from $(2.9)-(2.11)$ is completely continuous for fixed $\gamma$ and $\epsilon$, i.e., if $\left\{u_{k}\right\}$ converges weakly in $H^{1}(\Omega)$ to some $\bar{u} \in H^{1}(\Omega)$, then $p_{\gamma, \epsilon}\left(u_{k}\right)$ converges strongly to $p_{\gamma, \epsilon}(\bar{u})$ in $H_{0}^{1}(\Omega)$. See Appendix B for the proof.

Summarizing the above findings when passing to the limit as $\gamma \rightarrow \infty$ and $\epsilon \rightarrow 0$, we have the following stationarity conditions.

THEOREM 2.1. Assume that $\left\{\left(y_{\gamma_{k}, \epsilon_{k}}^{*}, u_{\gamma_{k}, \epsilon_{k}}^{*}\right)\right\}$ converges strongly-weakly in $H_{0}^{1}(\Omega) \times H^{1}(\Omega)$ to $\left(y^{*}, u^{*}\right) \in H_{0}^{1}(\Omega) \times H^{1}(\Omega)$ as $\gamma_{k} \rightarrow \infty$ and $\epsilon_{k} \rightarrow 0$. Then there exist a subsequence $\{k(l)\}$ and multipliers $\left(p^{*}, \mu^{*}, \lambda^{*}\right) \in H_{0}^{1}(\Omega) \times H^{-1}(\Omega) \times H^{-1}(\Omega)$ such that $\left\{\left(p_{\gamma_{k(l)}, \epsilon_{k(l)}}^{*}, \mu_{\gamma_{k(l)}, \epsilon_{k(l)}}^{*}, \lambda_{\gamma_{k(l)}, \epsilon_{k(l)}}^{*}\right)\right\}$ converges weakly in $H_{0}^{1}(\Omega) \times H^{-1}(\Omega) \times H^{-1}(\Omega)$ to $\left(p^{*}, \mu^{*}, \lambda^{*}\right)$ as $l \rightarrow \infty$ and

$$
\begin{aligned}
a\left(y^{*}, v ; u^{*}\right)-\left\langle\lambda^{*}, v\right\rangle-\left(f\left(u^{*}\right), v\right) & =0 \quad \forall v \in H_{0}^{1}(\Omega), \\
y^{*} \in K, \quad\left\langle\lambda^{*}, y^{*}\right\rangle=0, \quad\left\langle\lambda^{*}, v\right\rangle \geq 0 & \forall v \in K, \\
\left(y^{*}-y_{m}, v\right)+a\left(p^{*}, v ; u^{*}\right)+\left\langle\mu^{*}, v\right\rangle & =0 \quad \forall v \in H_{0}^{1}(\Omega), \\
\left\langle\alpha u^{*}+a_{u}\left(y^{*}, p^{*} ; u^{*}\right)-B^{*} p^{*}, w-u^{*}\right\rangle_{H^{1}(\Omega)^{\prime}, H^{1}(\Omega)} \geq 0 \quad \forall w \in U_{a d}, & \\
\left\langle y^{*}, \mu^{*}\right\rangle & =0, \\
\lim _{k \rightarrow \infty}\left\langle p_{\gamma_{k}, \epsilon_{k}}^{*}, \lambda_{\gamma_{k}, \epsilon_{k}}^{*}\right\rangle & =0, \\
\liminf _{k \rightarrow \infty}\left\langle p_{\gamma_{k}, \epsilon_{k}}^{*}, \mu_{\gamma_{k}, \epsilon_{k}}^{*}\right\rangle & \geq 0 .
\end{aligned}
$$

Following the terminology of mathematical programs with equilibrium constraints (see, e.g., [25]) we refer to $\left(y^{*}, u^{*}, \lambda^{*}\right)$ as a $C$-stationary point of $(\mathrm{P})$.

3. An active set type algorithm for the discrete problem. We proceed by introducing an active set type solver leading to a sequence of equality constrained approximations of $(\mathrm{P})$, i.e., the inequalities for $y$ and $\lambda$ are taken care of by active and inactive set estimates, respectively. Further, in every iteration feasibility of the primal iterates $\left(y_{k}, u_{k}, \lambda_{k}\right)$ is restored. Note that we have $y\left(u_{k}\right) \in W_{0}^{1, r}(\Omega)$ for some $r>2$. For $\Omega \subset \mathbb{R}^{2}$ Sobolev embedding yields $W_{0}^{1, r}(\Omega) \subset \mathcal{C}(\bar{\Omega})$. This fact supports a discretization of $\mu^{*}$ which is concentrated at the nodal points of a mesh $\Omega_{h} \subset \Omega$ with $h$ indicating the mesh size of discretization; similarly for $\lambda^{*}$. Utilizing a finite difference discretization of the system of Theorem 2.1 leads to

$$
\begin{aligned}
& A_{h}\left(u_{h}^{*}\right) y_{h}^{*}-\lambda_{h}^{*}-f_{h}\left(u_{h}^{*}\right)=0, \\
& \Theta\left(y_{h}^{*}, \lambda_{h}^{*}\right)=0, \\
& J_{h, y}\left(y_{h}^{*}, u_{h}^{*}\right)+A_{h}\left(u_{h}^{*}\right)^{\top} p_{h}^{*}+\mu_{h}^{*}=0, \\
&\left(J_{h, u}\left(y_{h}^{*}, u_{h}^{*}\right)+\left(y_{h}^{*}\right)^{\top} A_{h}^{\prime}\left(u_{h}^{*}\right)(\cdot)^{\top} p_{h}^{*}-B_{h}^{\top} p_{h}^{*}, u_{h}-u_{h}^{*}\right)_{h} \geq 0 \quad \forall u_{h} \in U_{\mathrm{ad}}^{h}, \\
& y_{h}^{*} \star \mu_{h}^{*}=0, \\
& p_{h}^{*} \star \lambda_{h}^{*}=0 .
\end{aligned}
$$

Above we have $y_{h}^{*}, p_{h}^{*}, \lambda_{h}^{*}, \mu_{h}^{*} \in \mathbb{R}^{n_{h}^{y}}$ and $u_{h}^{*} \in \mathbb{R}^{n_{h}^{u}}$ and further $A_{h}(\cdot) \in \mathbb{R}_{h}^{n_{h}^{y} \times n_{h}^{y}}, B_{h} \in \mathbb{R}^{n_{h}^{y} \times n_{h}^{u}}$. By $A_{h}^{\prime}\left(u_{h}\right)(\cdot)$ we denote the derivative of $A_{h}\left(u_{h}\right)$ with respect to $u_{h}$. Moreover, $\star: \mathbb{R}_{h}^{n_{h}^{y}} \times \mathbb{R}^{n_{h}^{y}} \rightarrow \mathbb{R}^{n_{h}^{y}}$ 
denotes the Hadamard product of vectors, $(\cdot, \cdot)_{h}$ is the discrete version of $\langle\cdot, \cdot\rangle_{H^{1}(\Omega)^{\prime}, H^{1}(\Omega)}$ and $\Theta\left(y_{h}, \lambda_{h}\right)=\left(\theta\left(y_{h, 1}, \lambda_{h, 1}\right), \ldots, \theta\left(y_{h, n_{h}^{y}}, \lambda_{h, n_{h}^{y}}\right)\right)$ with

$$
\theta(a, b)=0 \quad \Longleftrightarrow \quad a \geq 0, \quad b \geq 0, \quad a b=0
$$

for $a, b \in \mathbb{R}$. A particular instance of such a function is $\theta(a, b)=a-\max (0, a-\sigma b)$ for arbitrarily fixed $\sigma>0$.

In the algorithm below the variational inequality in (3.4) is treated by an interior point approach (see, e.g., [26] for an account of primal-dual interior-point methods). It computes a sequence of vectors $\left\{u_{h}^{(k)}\right\}$ which satisfies $\epsilon_{l}<u_{h, i}^{(k)}<\epsilon_{u}$ for all $i \in\left\{1, \ldots, n_{h}^{u}\right\}$ and for all $k$. The bounds may only be approached as $k \rightarrow \infty$. Hence, the interior point method acts as a regularization technique which allows us to replace (3.4) by

$$
\begin{aligned}
J_{h, u}\left(y_{h}, u_{h}\right)+\left(y_{h}\right)^{\top} A_{h}^{\prime}\left(u_{h}\right)(\cdot)^{\top} p_{h}-B_{h}^{\top} p_{h}-q_{l, h}+q_{u, h} & =0, \\
q_{l, h} \star\left(u_{h}-\epsilon_{l} e\right) & =\kappa e, \\
q_{u, h} \star\left(\epsilon_{u} e-u_{h}\right) & =\kappa e,
\end{aligned}
$$

where $e:=(1, \ldots, 1) \in \mathbb{R}^{n_{h}^{u}}$ and $q_{l, h}, q_{u, h} \in \mathbb{R}^{n_{h}^{u}}$ with $q_{l, h}, q_{u, h}>0$; see Appendix C for the socalled barrier problem leading to (3.7)-(3.9). Moreover, $\kappa>0$ denotes a so-called duality measure with the property that a solution of the interior-point-regularized system approaches a solution of (3.1)-(3.6) as $\kappa \rightarrow 0$.

3.1. The algorithm. Now we are ready to define the algorithm. In what follows, for ease of notation we dismiss the subscript $h$.

Algorithm.

(i) Initialization. Choose $u^{(0)}, p^{(0)}$ and $q_{l}^{(0)}, q_{u}^{(0)}$ such that $\epsilon_{l}<u_{i}^{(0)}<\epsilon_{u}$ and $q_{l, i}^{(0)}>0, q_{u, i}^{(0)}>0$ for all $i=1, \ldots, n_{h}^{u}$. Set $\kappa^{(0)}:=\max \left(\left(u^{(0)}-\epsilon_{l} e\right)^{\top} q_{l}^{(0)},\left(\epsilon_{u} e-u^{(0)}\right)^{\top} q_{u}^{(0)}\right)$. Choose $\nu \in(0,1)$, $\zeta \in(0,1)$, and set $k:=0$.

(ii) Feasibility restoration. Find the solution $\left(y^{(k)}, \lambda^{(k)}\right)$ of

$$
\begin{gathered}
A\left(u^{(k)}\right) y-\lambda=f\left(u^{(k)}\right), \\
\Theta(y, \lambda)=0 .
\end{gathered}
$$

(iii) Unless some stopping rule is satisfied, determine the inactive and active set estimates

$$
\mathcal{I}^{(k)}:=\left\{i: y_{i}^{(k)}>0\right\} \quad \text { and } \quad \mathcal{A}^{(k)}:=\left\{1, \ldots, n_{h}^{y}\right\} \backslash \mathcal{I}^{(k)} .
$$

(iv) Compute the update step $\left(d u^{(k)}, d y^{(k)}, d p^{(k)}, d q_{l}^{(k)}, d q_{u}^{(k)}\right)$ such that

$$
\begin{gathered}
\left(A\left(u^{(k)}\right) d y^{(k)}+\left[A^{\prime}\left(u^{(k)}\right)(\cdot) y^{(k)}-f^{\prime}\left(u^{(k)}\right)\right] d u^{(k)}\right)_{i}=\left(r_{1}^{(k)}\right)_{i} \quad \forall i \in \mathcal{I}^{(k)}, \\
d y_{i}^{(k)}=0 \quad \forall i \in \mathcal{A}^{(k)}, \\
\left(A\left(u^{(k)}\right)^{\top} d p^{(k)}+\left[A^{\prime}\left(u^{(k)}\right)(\cdot)^{\top} p^{(k)}\right] d u^{(k)}+J_{y y}\left(y^{(k)}, u^{(k)}\right) d y^{(k)}\right)_{i}=\left(r_{2}^{(k)}\right)_{i} \quad \forall i \in \mathcal{I}^{(k)}, \\
p_{i}^{(k)}+d p_{i}^{(k)}=0 \quad \forall i \in \mathcal{A}^{(k)},
\end{gathered}
$$




$$
\begin{aligned}
{\left[J_{u u}\left(y^{(k)}, u^{(k)}\right)+\left(p^{(k)}\right)^{\top} A^{\prime \prime}\left(u^{(k)}\right)(\cdot) y^{(k)}\right] d u^{(k)}+\left(A^{\prime}\left(u^{(k)}\right)(\cdot) y^{(k)}\right.} & \left.-f^{\prime}\left(u^{(k)}\right)\right)^{\top} d p^{(k)} \\
+\left(p^{(k)}\right)^{\top} A^{\prime}\left(u^{(k)}\right)(\cdot) d y^{(k)}-d q_{l}^{(k)}+d q_{u}^{(k)} & =r_{3}^{(k)}, \\
\left(u^{(k)}-\epsilon_{l} e\right) \star d q_{l}^{(k)}+q_{l}^{(k)} \star d u^{(k)} & =r_{4}^{(k)}, \\
\left(\epsilon_{u} e-u^{(k)}\right) \star d q_{u}^{(k)}+q_{u}^{(k)} \star d u^{(k)} & =r_{5}^{(k)},
\end{aligned}
$$

with the residuals

$$
\begin{aligned}
r_{1}^{(k)} & =f\left(u^{(k)}\right)-A\left(u^{(k)}\right) y^{(k)}, \\
r_{2}^{(k)} & =-J_{y}\left(y^{(k)}, u^{(k)}\right)-A\left(u^{(k)}\right)^{\top} p^{(k)}, \\
r_{3}^{(k)} & =f^{\prime}\left(u^{(k)}\right)^{\top} p^{(k)}-J_{u}\left(y^{(k)}, u^{(k)}\right)-\left(y^{(k)}\right)^{\top} A^{\prime}\left(u^{(k)}\right)(\cdot)^{\top} p^{(k)}+q_{l}^{(k)}-q_{u}^{(k)}, \\
r_{4}^{(k)} & =\kappa^{(k)} e-\left(u^{(k)}-\epsilon_{l} e\right) \star q_{l}^{(k)}, \\
r_{5}^{(k)} & =\kappa^{(k)} e-\left(\epsilon_{u} e-u^{(k)}\right) \star q_{u}^{(k)} .
\end{aligned}
$$

(v) Step. Determine the updates

$$
\begin{aligned}
& u^{(k+1)}:=u^{(k)}+s^{(k)} d u^{(k)}, \\
& p^{(k+1)}:=p^{(k)}+s^{(k)} d p^{(k)}, \\
& q_{u}^{(k+1)}:=q_{u}^{(k)}+s^{(k)} d q_{u}^{(k)}, \\
& q_{l}^{(k+1)}:=q_{l}^{(k)}+s^{(k)} d q_{l}^{(k)},
\end{aligned}
$$

where the step length $s^{(k)}>0$ is computed by

$$
s^{(k)}:=\min \left(1, \zeta \min \left(s_{l}^{(k)}, s_{u}^{(k)}, t_{l}^{(k)}, t_{u}^{(k)}\right)\right)
$$

with

$$
s_{u}^{(k)}:=\left\{\begin{array}{l}
\min \left(-\frac{q_{u, i}^{(k)}}{d q_{u, i}^{(k)}}: i \in \mathcal{N}_{q_{u}}^{(k)}:=\left\{i: d q_{u, i}^{(k)}<0\right\}\right) \text { if } \mathcal{N}_{q_{u}}^{(k)} \neq \emptyset \\
+\infty \text { otherwise }
\end{array}\right.
$$

and

$$
t_{u}^{(k)}:=\left\{\begin{array}{l}
\min \left(\frac{\epsilon_{u}-u_{i}^{(k)}}{d u_{i}^{(k)}}: i \in \mathcal{N}_{\epsilon_{u}}^{(k)}:=\left\{i: d u_{i}^{(k)}>0\right\}\right) \text { if } \mathcal{N}_{\epsilon_{u}}^{(k)} \neq \emptyset \\
+\infty \text { otherwise }
\end{array}\right.
$$

and analogously for $s_{l}^{(k)}$ and $t_{l}^{(k)}$ with $\mathcal{N}_{q_{l}}^{(k)}:=\left\{i: d q_{l, i}^{(k)}<0\right\}$ and $\mathcal{N}_{\epsilon_{l}}^{(k)}:=\left\{i: d u_{i}^{(k)}<0\right\}$, respectively. Set $\kappa^{(k+1)}:=\nu \kappa^{(k)}, k:=k+1$, and continue with step (ii).

In numerical practice, typical values for $\nu$ and $\zeta$ are $\nu=0.1$ and $\zeta=0.99$.

3.2. Discussion of the algorithm and convergence considerations. Next we address the various steps of the above algorithm. In fact, it turns out that the feasibility restoration phase consists of solving an obstacle-type problem in every iteration. Step (iv) corresponds to a Newton step for a particular equality constrained problem and is related to implicit programming techniques in MPECs $[18,19]$. Further, the step size selection used in (v) reflects the fraction-to-the-boundaryrule well-known from interior point methods; see, e.g., [26]. 
3.3. Discussion of the algorithm. Step (i). The initializations of $u$ and $q_{u}, q_{l}$ follow the usual interior point concept. All quantities are best initialized safely away from the boundaries of the respective feasible sets. Due to the identification context for $u$, choosing $u^{(0)}$ larger than the expected value for the parameter typically results in a more stable numerical behavior; otherwise, if $u$ gets too close to $\epsilon_{l}$ (for small $\epsilon_{l}$ ), $A(u)$ becomes nearly singular which implies reduced numerical stability of the identification process. The initial choice of $\kappa$ again is related to the usual choice in the context of interior point methods; see, e.g., [26].

Step (ii). We assume that the discretization yields a symmetric and, due to $u^{(k)} \geq \epsilon_{l}>0$, positive definite matrix $A\left(u^{(k)}\right)$. Then the system of step (ii) is the first order necessary and sufficient optimality condition of the quadratic convex minimization problem

$$
\begin{aligned}
& \text { minimize } \frac{1}{2} y^{\top} A\left(u^{(k)}\right) y-\left(f\left(u^{(k)}\right)^{\top} y \text { over } y \in \mathbb{R}^{n_{h}^{y}}\right. \\
& \text { subject to } y \geq 0 .
\end{aligned}
$$

The complementarity system arising due to the inequality constraints, i.e.,

$$
y^{(k)} \geq 0, \quad \lambda^{(k)} \geq 0, \quad\left(y^{(k)}\right)^{\top} \lambda^{(k)}=0,
$$

is equivalent to

$$
\Theta\left(y^{(k)}, \lambda^{(k)}\right)=0 .
$$

Note that step (ii) restores primal feasibility of the iterate $\left(y^{(k)}, u^{(k)}, \lambda^{(k)}\right)$. In the continuous setting, (3.10) corresponds to an obstacle problem [23]. For its numerical solution we use a semismooth Newton solver [11, 12], but alternative techniques such as monotone multigrid methods $[13,17]$ may be invoked as well; see [7] for further techniques. Our semismooth Newton solver converges globally with a locally superlinear rate.

Step (iii). The main purpose of this step is a careful treatment of (3.5)-(3.6). In fact, note that $y_{i}^{*}>0$ implies $\mu_{i}^{*}=0$ as well as $\lambda_{i}^{*}=0$ and $\lambda_{i}^{*}>0$ implies $p_{i}^{*}=0$. These relations are used in the subsequent step. In our implementation we stop the algorithm as soon as the residual of (3.1)-(3.6) at iteration $k$, denoted by res ${ }^{(k)}$, satisfies

$$
\left\|\operatorname{res}^{(k)}\right\| \leq \epsilon_{\text {tol }}\left(1+\| \text { res }^{(0)} \|\right)
$$

for some user-specified tolerance $0<\epsilon_{t o l} \ll 1$.

Step (iv). This step corresponds to a linearization of the first order optimality system taking into account the active and inactive set estimates of step (iii). Indeed, the first two equations in step (iv) correspond to a generalized linearization of

$$
A(u) y-\lambda-f(u)=0 \quad \text { and } \quad \Theta(y, \lambda)=0 \quad \text { at }\left(y^{(k)}, u^{(k)}\right)
$$

with $\Theta(y, \lambda)=\lambda-\max (0, \lambda-\sigma y)$. Recall from step (iii) that we expect $\lambda_{i}^{(k+1)}=\lambda_{i}^{(k)}+d \lambda_{i}^{(k)}=0$ for $i \in \mathcal{I}^{(k)}$ and, further, $y_{i}^{(k+1)}=y_{i}^{(k)}+d y_{i}^{(k)}=0$ for $i \in \mathcal{A}^{(k)}$. Thus, linearizing (3.1) and considering only indices in $\mathcal{I}^{(k)}$ yields

$$
\left(A\left(u^{(k)}\right)\left(y^{(k)}+d y\right)+A^{\prime}\left(u^{(k)}\right)(d u) y^{(k)}-f\left(u^{(k)}\right)-f^{\prime}\left(u^{(k)}\right) d u\right)_{i}=0 \quad \forall i \in \mathcal{I}^{(k)}
$$

which corresponds to the first equation in step (iv). The second equation results from $y_{i}^{(k+1)}=$ $y_{i}^{(k)}=0$ for $i \in \mathcal{A}^{(k)}$. Next we introduce the set

$$
\mathcal{L}^{(k)}:=\left\{i: \lambda_{i}^{(k)}>0\right\} \subset \mathcal{A}^{(k)}
$$


and assume that strict complementarity holds true at iteration $k$, i.e., $\mathcal{A}^{(k)}=\mathcal{L}^{(k)}$. Concerning the third and the forth equation of step (iv) we first recall that $\mu_{i}^{(k+1)}=\mu_{i}^{(k)}+d \mu_{i}^{(k)}=0$ is expected for all $i \in \mathcal{I}^{(k)}$. Then a similar reasoning as before yields

$$
\left(y^{(k)}+d y-y_{m}+A\left(u^{(k)}\right)^{\top}\left(p^{(k)}+d p\right)+A^{\prime}\left(u^{(k)}\right)(d u)^{\top} p^{(k)}\right)_{i}=0 \quad \forall i \in \mathcal{I}^{(k)},
$$

which is the third equation. The forth equation results from $p_{i}^{(k+1)}=p_{i}^{(k)}+d p_{i}^{(k)}=0$ for all $i \in \mathcal{I}^{(k)}$. The final three equations of step (iv) arise from a straightforward linearization of (3.7)-(3.9) at $\left(y^{(k)}, u^{(k)}, \lambda^{(k)}, p^{(k)}, q_{u}^{(k)}, q_{l}^{(k)}\right)$.

Step (v). The step size selection reflects the fraction-to-the-boundary-rule of interior point methods. Below we argue exemplarily for $q_{u}$; the other variables and bounds are treated analogously. At iteration $k$ we have $q_{u}^{(k)}>0$. For indices with $d q_{u, i}^{(k)} \geq 0$ we have $q_{u, i}^{(k)}+s d q_{u, i}^{(k)} \geq q_{u, i}^{(k)}>0$ for all $s>0$. Hence, no restriction on $s$ arises. On the other hand, for $d q_{u, i}^{(k)}<0$ we find that for $s_{u, i}^{(k)}=-q_{u, i}^{(k)} / d q_{u, i}^{(k)}>0$ we have

$$
q_{u, i}^{(k)}+s_{u, i}^{(k)} d q_{u, i}^{(k)}=0 \quad \text { and } \quad q_{u, i}^{(k)}+\zeta s_{u, i}^{(k)} d q_{u, i}^{(k)}>0 \text { for } \zeta \in(0,1)
$$

Taking $s_{u}^{(k)}=\min \left(s_{u, i}^{(k)}: i\right.$ with $\left.d q_{u, i}^{(k)}<0\right)$ guarantees $q_{u}^{(k+1)}=q_{u}^{(k)}+\zeta s_{u}^{(k)} d q_{u}^{(k)}>0$, i.e., the next iterate stays away from the boundary.

3.4. Convergence considerations. Now we related our algorithm to implicit programming (IP) methods in MPECs. The IP technique reduces the discrete version of our model problem (P) to

$$
\text { minimize } J(y(u), u) \text { s.t. } u \in U_{\mathrm{ad}} \subset \mathbb{R}^{n_{h}^{u}}
$$

where $y(u) \in \mathbb{R}^{n_{h}^{y}}$ is the unique solution of the discrete linear complementarity problem

$$
A(u) y-\lambda=f(u), \quad y \geq 0, \quad \lambda \geq 0, \quad y^{\top} \lambda=0
$$

This problem is the first order optimality system of (3.10) at $u$. Next we study smoothness properties of $y(u)$. First note that $F(y, u):=A(u) y-f(u)$ is continuously differentiable with respect to $y$ and $u$. Define the lower level constraint set $C=\left\{y \in \mathbb{R}_{h}^{n_{h}}: g(y) \leq 0\right\}$ with $g(y)=-y$. Then for arbitrary $\mathcal{S} \subset\left\{1, \ldots, n_{h}^{y}\right\}$ the vector $v=(1, \ldots, 1)^{\top} \in \mathbb{R}_{h}^{n_{h}^{y}}$ satisfies

$$
\nabla g_{i}(y)^{\top} v=-1<0
$$

Hence, we say that the Mangasarian-Fromovitz constraint qualification holds true at any $y$. Further, the rank of the family of gradient vectors $\left\{\nabla g_{i}(y): i \in \mathcal{A}\right\}$ with $\mathcal{A} \subset \mathcal{A}(y(u)):=\left\{i: y(u)_{i}=0\right\}$ for arbitrary $y \in \mathbb{R}^{n_{h}^{y}}$ is a constant only depending on $\mathcal{A}$. Thus, the constant rank constraint qualification is said to hold true. Note that the pair $(y(u), \lambda(u))$ satisfying $(3.13)$ is unique. Now we introduce the VI-Lagrangian

$$
L(u, y, \lambda)=A(u) y-f(u)-\lambda,
$$

and we define the matrix

$$
\Lambda:=\left(\begin{array}{cc}
\nabla_{y} L(u, y(u), \lambda(u)) & \nabla g_{\mathcal{A}(y(u))}(y(u))^{\top} \\
-\nabla g_{\mathcal{A}(y(u))}(y(u)) & 0
\end{array}\right)=\left(\begin{array}{cc}
A(u) & E_{\mathcal{A}(y(u))}^{\top} \\
-E_{\mathcal{A}(y(u))} & 0
\end{array}\right)
$$


where $E_{\mathcal{A}(y(u))} \in \mathbb{R}^{|\mathcal{A}(y(u))| \times n_{h}^{y}}$ contains in each row an element $e_{i, l}=-1$ and otherwise $e_{i, j}=0$ for $j \neq l$. Hence, $E_{\mathcal{A}(y(u))}$ has full rank. Since $A(u)$ is nonsingular, so is $\Lambda$. Therefore, the strong coherent orientation condition is said to hold true. Summarizing, we have just shown that the assumptions of [18, Thm. 4.2.16] are satisfied. Then, according to this theorem, there exists a piecewise $C^{1}$-function $y(\cdot)$ in a neighborhood $U \times Y$ of $(u, y(u))$ such that $y(v) \in Y$, with $v \in U$, is the unique solution of (3.13) for $u=v$. For the definition of piecewise $C^{1}$-functions we refer to [18, Def. 4.2.7]. It is well-known that piecewise $C^{1}$-functions do not need to be Frechet-differentiable; see, e.g., [22]. From this we conclude that (3.12) is a nonsmooth (i.e., nondifferentiable) and nonconvex minimization problem, in general.

For a perturbation direction $d u \in \mathbb{R}_{h}^{n_{h}^{u}}$ let $y^{\prime}\left(u^{(k)} ; d u\right)$ denote the directional derivative of $y(\cdot)$ at $u^{(k)}$ in direction $d u$. Then, at iteration $k$ of an IP method the following auxiliary problem has to be solved for obtaining an update direction $d u^{(k)}$ :

$$
\begin{aligned}
& \operatorname{minimize}\left(w^{(k)}\right)^{\top} d u+\left(z^{(k)}\right)^{\top} y^{\prime}\left(u^{(k)} ; d u\right)+\frac{1}{2} d u^{\top} W^{(k)} d u \\
& \text { subject to } u^{(k)}+d u \in U_{\mathrm{ad}},
\end{aligned}
$$

where $w^{(k)}:=J_{u}\left(y^{(k)}, u^{(k)}\right)$ and $z^{(k)}:=J_{y}\left(y^{(k)}, u^{(k)}\right)$, with $y^{(k)}:=y\left(u^{(k)}\right)$. Further, $W^{(k)}$ is a symmetric and positive definite $n_{h}^{u} \times n_{h}^{u}$-matrix. In order to related (3.14) to step (iv) and in connection with solution procedures for $(3.14)$ we have to study $y^{\prime}\left(u^{(k)} ; d u\right)$. For this purpose we first define the biactive set at $u^{(k)}$ by

$$
\mathcal{B}^{(k)}:=\left\{i \in \mathcal{A}^{(k)}: \lambda_{i}^{(k)}=0\right\},
$$

and then we denote by

$$
\mathcal{K}\left(u^{(k)}, \lambda^{(k)} ; d u\right):=\left\{d y \in \mathbb{R}^{n_{h}^{y}}: d y_{i} \geq 0 \forall i \in \mathcal{B}^{(k)}, d y_{i}=0 \forall i \in \mathcal{L}^{(k)}\right\}
$$

the directional critical set at $u^{(k)}$ along $d u$. Then, according to [18, Thm. 4.2.25], $d y_{p}^{(k)}:=$ $y^{\prime}\left(u^{(k)} ; d u\right) \in \mathcal{K}\left(u^{(k)}, \lambda^{(k)} ; d u\right)$ is the unique solution of the affine variational inequality

$$
\left(d y-d y_{p}^{(k)}\right)^{\top}\left(\left[A^{\prime}\left(u^{(k)}\right)(\cdot) y^{(k)}-f^{\prime}\left(u^{(k)}\right)\right] d u+A\left(u^{(k)}\right)\right) \geq 0 \quad \forall d y \in \mathcal{K}\left(u^{(k)}, \lambda^{(k)} ; d u\right) .
$$

Note that (3.15) represents the first order necessary and sufficient condition of the convex quadratic problem

$$
\begin{array}{ll}
\operatorname{minimize} & \frac{1}{2} d y_{\mathcal{N}^{(k)}}^{\top} A_{\mathcal{N}^{(k)}}^{(k)} d y_{\mathcal{N}^{(k)}}+\left(\ell_{\mathcal{N}^{(k)}}^{(k)}\right)^{\top} d y_{\mathcal{N}^{(k)}} \text { over } d y_{\mathcal{N}^{(k)}} \in \mathbb{R}^{\left|\mathcal{N}^{(k)}\right|} \\
\text { subject to } & d y_{\mathcal{B}^{(k)}} \geq 0
\end{array}
$$

with

$$
\begin{aligned}
\mathcal{N}^{(k)} & :=\mathcal{I}^{(k)} \cup \mathcal{B}^{(k)}, \\
A_{\mathcal{N}^{(k)}}^{(k)} & :=\left(A\left(u^{(k)}\right)_{i j}\right) \quad \text { for } i, j \in \mathcal{N}^{(k)}, \\
\ell_{\mathcal{N}^{(k)}}^{(k)} & :=\left(\left[A^{\prime}\left(u^{(k)}\right)(\cdot) y^{(k)}-f^{\prime}\left(u^{(k)}\right)\right] d u\right)_{i} \quad \text { for } i \in \mathcal{N}^{(k)} .
\end{aligned}
$$

Recall that the biactive set $\mathcal{B}^{(k)}$ satisfies $\mathcal{B}^{(k)} \subset \mathcal{A}^{(k)}$. As for (3.10), for (3.15) there are very efficient solution procedures available. 
From now on let us assume that strict complementarity holds true at $u^{(k)}$, i.e., $\mathcal{A}^{(k)}=\mathcal{L}^{(k)}$ for all $k$. Then $\mathcal{B}^{(k)}=\emptyset,(3.16)$ becomes an unconstrained minimization problem and (3.15) is equivalent to

$$
\begin{aligned}
\left(\left[A^{\prime}\left(u^{(k)}\right)(\cdot) y^{(k)}-f^{\prime}\left(u^{(k)}\right)\right] d u+A\left(u^{(k)}\right) d y_{p}^{(k)}\right)_{i}=0 & \forall i \in \mathcal{I}^{(k)}, \\
\left(d y_{p}^{(k)}\right)_{i}=0 & \forall i \in \mathcal{A}^{(k)} .
\end{aligned}
$$

Due to the feasibility restoration in step (ii) of our algorithm and the equivalence of (3.10) and (3.11) at $u=u^{(k)}$ we have

$$
\left(r_{1}^{(k)}\right)_{i}=\left(f\left(u^{(k)}\right)-A\left(u^{(k)}\right) y^{(k)}\right)_{i}=\lambda_{i}^{(k)}=0 \quad \forall i \in \mathcal{I}^{(k)} .
$$

Hence, (3.17)-(3.18) together with (3.19) yield the first two equations of step (iv) of our algorithm. Thus, we conclude that, under our strict complementarity assumption at $u^{(k)}$, the Newton step computes $y^{\prime}\left(u^{(k)} ; d u\right)$, i.e., $d y^{(k)}=d y_{p}^{(k)}=y^{\prime}\left(u^{(k)} ; d u\right)$. The remaining equations of step (iv) serve the purpose of selecting a particular direction $d u$. This aspect is discussed next.

For the investigation concerning the determination of $d u=d u^{(k)}$ we neglect the interior point treatment of the inequalities for $u$. Rather we work in the framework of (3.12) with $u=u^{(k)}$. Due to our strict complementarity assumption [18, Prop. 4.2.30] yields Frechet-differentiability of $y(\cdot)$ at $u^{(k)}$. In this case, $d y_{p}^{(k)}=y^{\prime}\left(u^{(k)} ; d u\right)$ along with an appropriate $d \lambda^{(k)}$ is the unique solution of

$$
\begin{aligned}
A\left(u^{(k)}\right) d y-d \lambda & =\left[f^{\prime}\left(u^{(k)}\right)-A^{\prime}\left(u^{(k)}\right)(\cdot) y^{(k)}\right] d u, \\
d y_{i} & =0 \quad \forall i \in \mathcal{A}^{k}, \\
d \lambda_{i} & =0 \quad \forall i \in \mathcal{I}^{k} .
\end{aligned}
$$

Next choose $\tilde{W}^{(k)} \in \mathbb{R}^{\left(n_{h}^{y}+n_{h}^{u}\right) \times\left(n_{h}^{y}+n_{h}^{u}\right)}$ by

$$
\tilde{W}^{(k)}:=\left(\begin{array}{cc}
J_{y y}\left(y^{(k)}, u^{(k)}\right) & A^{\prime}\left(u^{(k)}\right)(\cdot)^{\top} p^{(k)} \\
\left(p^{(k)}\right)^{\top} A^{\prime}\left(u^{(k)}\right)(\cdot) & J_{u u}\left(y^{(k)}, u^{(k)}\right)+\left(p^{(k)}\right)^{\top} A^{\prime \prime}\left(u^{(k)}\right)(\cdot) y^{(k)}
\end{array}\right),
$$

and consider (3.14) under our assumptions, i.e.,

$$
\begin{aligned}
& \operatorname{minimize}\left(w^{(k)}\right)^{\top} d u+\left(z^{(k)}\right)^{\top} d y+\frac{1}{2}\left(d y^{\top}, d u^{\top}\right) \tilde{W}^{(k)}\left(\begin{array}{l}
d y \\
d u
\end{array}\right) \\
& \text { over }(d y, d u, d \lambda) \in \mathbb{R}_{h}^{n_{h}^{y}} \times \mathbb{R}^{n_{h}^{u}} \times \mathbb{R}^{n_{h}^{y}} \text {, } \\
& \text { subject to } u^{(k)}+d u \in U_{\text {ad }} \text {, } \\
& A\left(u^{(k)}\right) d y-d \lambda=\left[f^{\prime}\left(u^{(k)}\right)-A^{\prime}\left(u^{(k)}\right)(\cdot) y^{(k)}\right] d u, \\
& d y_{i}=0 \quad \forall i \in \mathcal{A}^{k} \text {, } \\
& d \lambda_{i}=0 \quad \forall i \in \mathcal{I}^{k} \text {. }
\end{aligned}
$$

We assume that the reduced Hessian of (3.24), which is obtained after eliminating $d y$, is positive definite. By $\left(\hat{q}^{(k+1)}, \hat{p}^{(k+1)}, r_{\mathcal{A}}^{(k)}, r_{\mathcal{I}}^{(k)}\right)$ we denote the multipliers associated with the constraints in (3.24). Further, let $E_{\mathcal{A}}^{(k)} \in \mathbb{R}^{n_{h}^{y} \times\left|\mathcal{A}^{(k)}\right|}$ denote the extension-by-zero operator of vectors in $\mathbb{R}^{\left|\mathcal{A}^{(k)}\right|}$ and analogously for $E_{\mathcal{I}}^{(k)} \in \mathbb{R}^{n_{h}^{y} \times\left|\mathcal{I}^{(k)}\right|}$. Then, by standard KKT-theory, the optimal so- 
lution $\left(\widehat{d y}^{(k)}, \widehat{d u}^{(k)}, \widehat{d \lambda}^{(k)}\right)$ of (3.24) is characterized by (primal) feasibility, the dual system

$$
\begin{aligned}
& J_{y y}\left(y^{(k)}, u^{(k)}\right) \widehat{d y}^{(k)}+\left[A^{\prime}\left(u^{(k)}\right)(\cdot)^{\top} p^{k}\right] d u+A\left(u^{(k)}\right)^{\top} \widehat{p}^{k+1}+E_{\mathcal{A}}^{(k)} r_{\mathcal{A}}^{(k)}=-z^{(k)}, \\
& \left(p^{(k)}\right)^{\top} A^{\prime}\left(u^{(k)}\right)(\cdot) \widehat{d y}^{(k)}+\left[J_{u u}\left(y^{(k)}, u^{(k)}\right)+\left(p^{(k)}\right)^{\top} A^{\prime \prime}\left(u^{(k)}\right)(\cdot) y^{(k)}\right] \widehat{d u}^{(k)} \\
& \quad+\left(A^{\prime}\left(u^{(k)}\right)(\cdot) y^{(k)}-f^{\prime}\left(u^{(k)}\right)\right)^{\top} \widehat{p}^{k+1}+\widehat{q}^{k+1}=-w^{(k)} \\
& -\widehat{p}^{k+1}+E_{\mathcal{I}}^{(k)} r_{\mathcal{I}}^{(k)}=0
\end{aligned}
$$

and a complementarity system involving $\widehat{q}^{(k+1)}$ and the constraints on $u^{(k)}+d u$. Now observe that the last three equations in the constraint set of (3.24) are equivalent to the first two equations of step (iv) in our algorithm. Setting $\widehat{p}^{(k+1)}=: p^{(k)}+d p^{(k)}$ we find from (3.27)

$$
\left(p^{(k)}+d p^{(k)}\right)_{i}=0 \quad \forall i \in \mathcal{A}^{(k)},
$$

which corresponds to the fourth equation of step (iv). The third equation is obtained from (3.25) and $\left(E_{\mathcal{A}}^{(k)} r_{\mathcal{A}}^{(k)}\right)_{i}=0$ for all $i \in \mathcal{I}^{(k)}$. Finally, replacing the interior point treatment by the usual multiplier approach for constraints of the type $u^{(k)}+d u^{(k)} \in U_{\text {ad }}$ we infer the fifth equation of step (iv). The remaining equations of step (iv) reflect the interior point methodology.

Summarizing, we find that step (iv) of our algorithm corresponds to the KKT-system of (3.24) with an interior point treatment for the box constraints on $u$. Hence, we conclude that our algorithm is tightly related to the implicit programming approach.

For a successful global convergence analysis of our algorithm, two ingredients are still missing: (i) A choice of the Hessian of the objective in (3.24) whenever the reduced Hessian of this problem is not positive definite; (ii) a line search procedure for globalization. With respect to (i) we note that in our setting a simple choice in case of lack of positive definiteness of the reduced Hessian based on $\tilde{W}^{(k)}$ in $(3.23)$ is given by

$$
\tilde{W}^{(k)}:=\left(\begin{array}{cc}
J_{y y}\left(y^{(k)}, u^{(k)}\right) & 0 \\
0 & J_{u u}\left(y^{(k)}, u^{(k)}\right)
\end{array}\right) .
$$

Of course, then step (iv) has to be change correspondingly. Concerning the line search globalization in (ii) raised above, the following procedure may be used: Let $\ell^{(k)} \in \mathbb{N}$ denote the smallest integer such that with $\tau=\omega^{\ell}$, for $\omega \in(0,1)$,

$$
\phi^{(k)}(\tau)-\phi^{(k)}(0) \leq \xi \tau\left(\phi^{(k)}\right)^{\prime}\left(0 ; d u^{(k)}\right),
$$

where $\xi \in(0,1)$ is fixed and

$$
\begin{aligned}
\phi^{(k)}(\tau) & :=J\left(y\left(u^{(k)}+\tau d u^{(k)}\right), u^{(k)}+\tau d u^{(k)}\right), \\
\left(\phi^{(k)}\right)^{\prime}\left(0 ; d u^{(k)}\right) & :=J_{u}\left(y^{(k)}, u^{(k)}\right)^{\top} d u^{(k)}+J_{y}\left(y^{(k)}, u^{(k)}\right)^{\top} y^{\prime}\left(u^{(k)} ; d u^{(k)}\right) .
\end{aligned}
$$

Then set $y^{(k+1)}:=y\left(u^{(k)}+\tau^{(k)} d u^{(k)}\right)$ and $u^{(k+1)}:=u^{(k)}+\tau^{(k)} d u^{(k)}$ with $\tau^{(k)}=\omega^{\ell^{(k)}}$.

Let $W^{(k)}$ denote the reduced Hessian obtained from (3.24) after eliminating $d y$. If there exist constants $0<c_{1} \leq c_{2}<+\infty$ such that

$$
c_{1}\|u\|^{2} \leq u^{\top} W^{(k)} u \leq c_{2}\|u\|^{2} \quad \forall k \in \mathbb{N},
$$


then, under all of the above assumptions, [18, Thm. 6.3.4] yields convergence of our algorithm (including the above line search globalization) to a stationary point satisfying (3.1)-(3.6).

In our numerical practice (see the next section) we find that the interior point version of our algorithm with the fraction-to-the-boundary line search and the choice (3.23) exhibits a convergent behavior, as well. Hence, in our numerics we dispense with the (expensive) line search procedure mentioned above. However, we emphasize that for a rigorous convergence proof the line search and the uniform positive definiteness of $\left\{\tilde{W}^{(k)}\right\}$ are needed.

4. Numerical results. In our subsequent test problems the coefficient $u$ is discretized on a coarser mesh than the state $y$ of the system. Typically we have $h_{u}=2 h_{y}$, where $h_{y}$ denotes the mesh size of the discretization of $y$ and $h_{u}$ is the one for $u$. This reduces the degrees of freedom for $u$ and stabilizes the identification process in the case of insufficient data or rather flat regions in $y$. We also note that further coarsening of the mesh for discretizing $u$ may be considered. It might also be interesting to explore locally refined/coarsened meshes. Moreover, observe that in contrast to inverse coefficient problems for partial differential equations the singular set

$$
\mathcal{S}_{0}=\{x \in \Omega: \nabla y(x)=0\},
$$

where the identification of $u$ is highly problematic, may be of large Lebesgue measure. In our numerics, based on the measurement data $y_{m}$ we estimate this set by a thresholding technique and do not allow degrees of freedom for $u$ in this set. In all cases reported below, the discretization of $A(u)$ is based on a standard box scheme.

Example 1. Our first example corresponds to a lubrication problem; see [4]. The data $y_{m}$ is obtained by solving the VI on a fine mesh with fixed $u$, i.e., $u=u_{f}=1+0.5 \cos \left(2 \pi x_{2}\right)$ on the domain $\Omega=(0,1)^{2}$ and restricting the fine grid solution to the coarse mesh for the identification. The coefficient function is $c(z)=z^{3}$, and the right hand side is $f(u)=\frac{\partial u}{\partial x_{2}}$. The regularization parameter is $\alpha=7.5 \mathrm{E}-3$, and a $H^{1}$-seminorm regularization is used, i.e., $R(u)=\|\nabla u\|_{L^{2}(\Omega)}^{2}$. The left plot in figure 4.1 shows the computational domain for $u$ in light gray which is estimated from the measurement $y_{m}$ shown in the right plot (note that dark gray corresponds to values of $|\nabla y(x)|$ close to zero). All other quantities, except for the multipliers corresponding to $u \in U_{\text {ad }}$, are discretized on the whole domain. The lower bound on $u$ is chosen to be $\epsilon_{l}=1 \mathrm{E}-6$ and the upper bound is
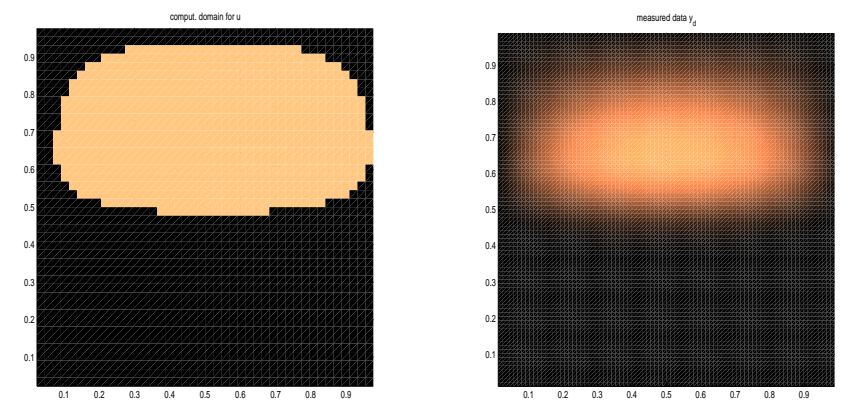

FIG. 4.1. Example 1. Computational domain for u (light color; left) estimated from measurement data (right).

$\epsilon_{u}=1$ E6. At the numerical solution none of the bounds is active. The algorithm is initialized by

$$
y^{(0)} \equiv 1, \quad u^{(0)} \equiv 10, \quad q_{l}^{(0)}=q_{u}^{(0)} \equiv 10, \quad \lambda^{(0)}=\mu^{(0)}=p^{(0)} \equiv 0 .
$$


We realize a nested iteration framework by employing a hierarchy of four grids with mesh-sizehalving for the refinement. The coarsest mesh size is $h_{y}^{(0)}=1 / 11$. We use the above initialization on the coarsest mesh only. The initial points on the finer meshes are obtained as follows: We solve the coarse mesh problem up to an accuracy of $\epsilon_{\text {tol }}=1 \mathrm{E}-8$. Then the coarse grid solution is prolongated to the next finer mesh to obtain the initial point on the fine mesh. This procedure is repeated until the finest mesh is reached.

In figure 4.2 we depict the numerical solution. The left plot shows the reconstructed parameter. Note that we extend the numerical solution on the computational domain (left side in the plot) for $u$ to the whole domain by the (known) true values. In the middle the state $y$ is shown and on the right $\lambda$ is plotted. We clearly see the complementary nature of $y$ and $\lambda$. In figure 4.3 we
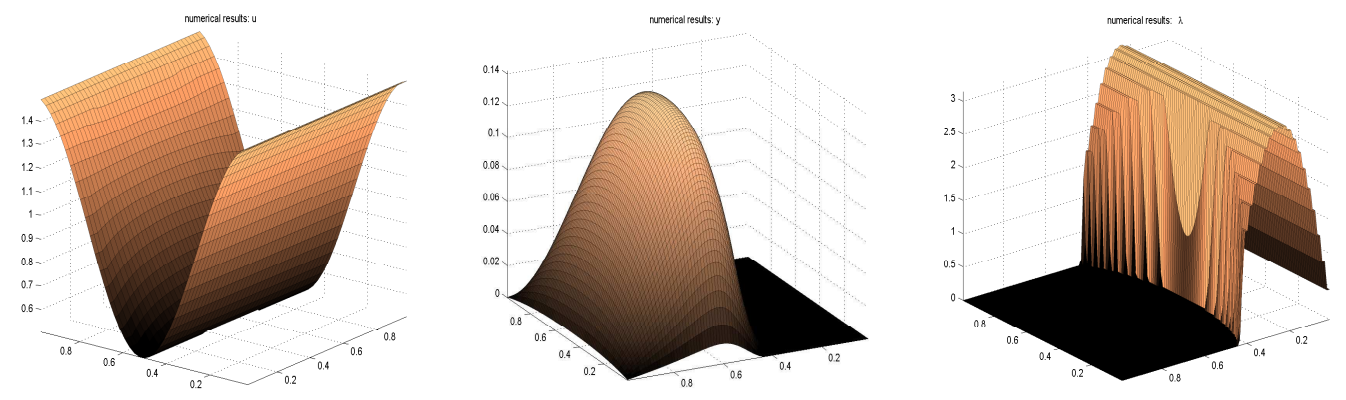

FIG. 4.2. Example 1. Reconstruction u (left), state $y$ (center) and corresponding multiplier $\lambda$ (right) at the numerical solution.

present the numerical convergence behavior on the finest mesh. The left plot shows the primal residual $\left\|A\left(u^{(k+1)}\right) y^{(k)}-\lambda^{(k)}-f\left(u^{(k+1)}\right)\right\|$ corresponding to $(3.1)$, and the right plot depicts the residual norm of the optimality with respect to $u$; cf. (3.4). In both cases, on the horizontal axis the iterations are plotted and on the vertical axis the respective residual norm is shown in a logarithmic scale. Starting from iteration 15 we clearly identify a superlinear convergence rate. The result for the adjoint equation is similar.
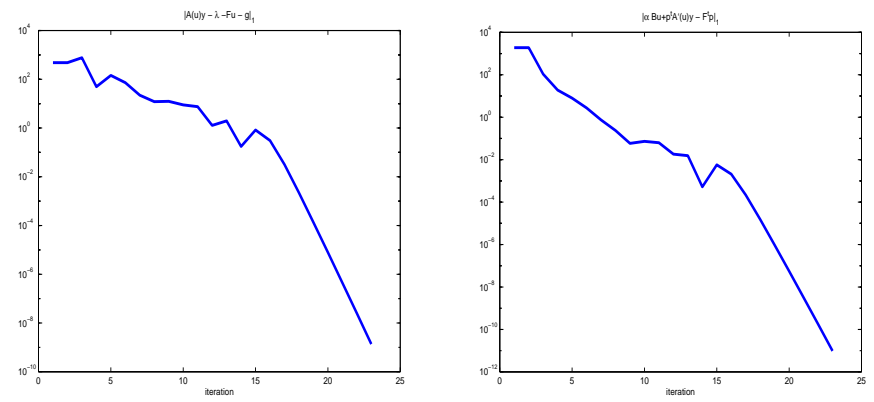

Fig. 4.3. Example 1. Convergence of residuals: Primal constraint (left) and optimality w.r.t. u (right).

Example 2. This example is challenging as the numerical solution exhibits a degenerated or biactive zone. Here we have $\alpha=5 \mathrm{E}-6, \Omega=(0,1)^{2}, c(z)=(z+0.1)^{2}+0.1$, and $f(u)=\left(x_{1}-\right.$ $0.5)\left(x_{2}-0.9\right)$. The bounds on $u$ are as before, with none of them active at the numerical solution. 
The meshes and the initialization on the coarsest mesh are as in example 1 . The fixed parameter $u_{f}$ is $u_{f}=x_{1}\left(1-x_{2}\right) x_{2}\left(1-x_{2}\right)$. In figure 4.4 we depict the computational domain for $u$ in the left plot. This domain is determined from $y_{m}$ in the right plot.
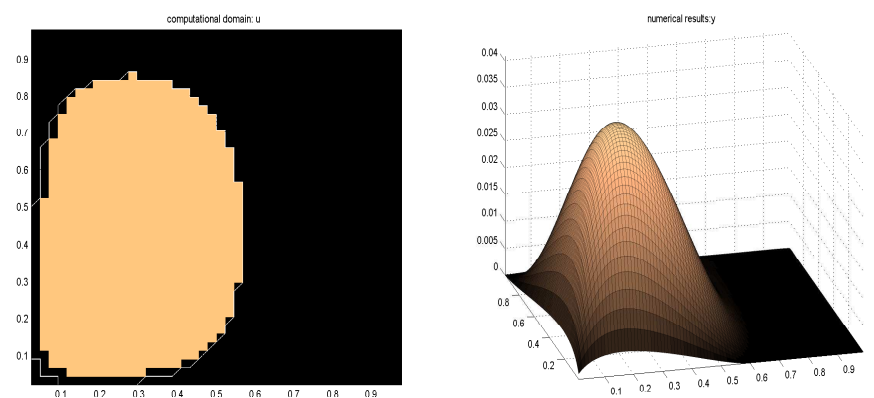

FIG. 4.4. Example 2. Computational domain for u (light color; left) estimated from measurement data $y_{m}$ (right).

The reconstruction $u$, again extended by the true value, is shown in the left plot of figure 4.5. The right plot depicts the multiplier $\lambda$ at the numerical solution.
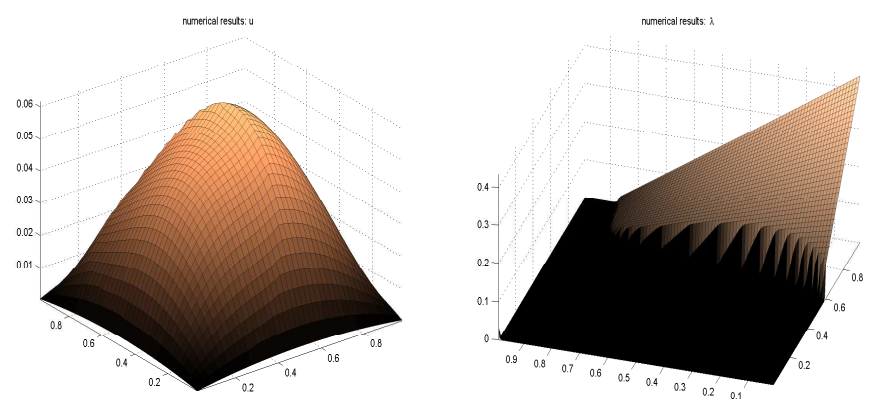

FIG. 4.5. Example 2. Reconstruction u (left) and corresponding multiplier $\lambda$ (right) at the numerical solution.

The degeneracy of $y$ and $\lambda$ can best be seen from figure 4.6, where the projections of $y$ and $\lambda$ onto the $x_{1} x_{2}$-plane are shown. Dark gray corresponds to very small or zero values of $y$ and $\lambda$. The state $y$ is shown in the left plot and $\lambda$ in the right. The region in the upper right corner, where $y \approx 0$ and $\lambda \approx 0$, reflects the high degeneracy of this test problem.

Similar as for example 1 we study the convergence speed of our algorithm by plotting the residuals of the primal equation and the optimality with respect to $u$; see figure 4.7. Again, the horizontal axes correspond to the iterations and the vertical axes show the respective residual in a logarithmic scale. Starting from iteration 17 a superlinear rate of convergence is identified. In conclusion, the numerical behavior of our method does not suffer from the adverse effect of degeneracy of this example.

The efficiency of the nested iteration concept is reflected in table 4.1, where the iteration number on the respective mesh is shown. Clearly, most iterations are spent on the coarse mesh, while the iteration counts on the fine meshes stay almost constant at a rather low level. The conclusions for example 1 are similar. 

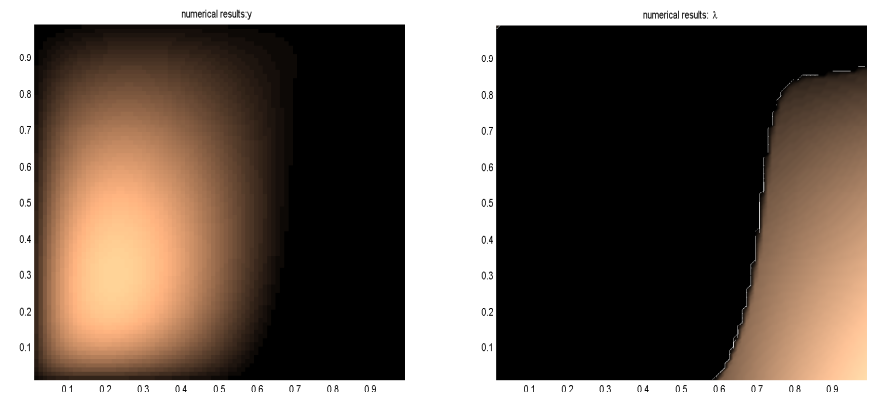

Fig. 4.6. Example 2. Projection onto the $x_{1} x_{2}$-plane of $y$ (left) and $\lambda$ (right) at the numerical solution.
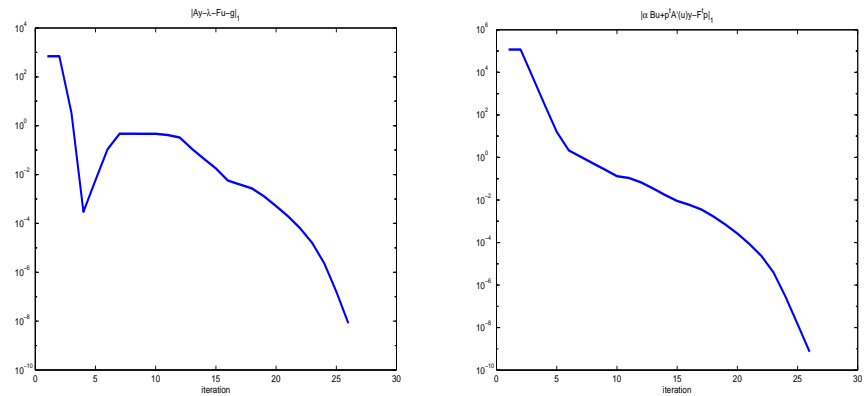

Fig. 4.7. Example 2. Convergence of residuals: Primal constraint (left) and optimality w.r.t. u (right).

We finally point out that in connection with MPECs one is ultimately interested in strong stationarity; see, e.g., [24] for its definition in finite dimensions. A rigorous theoretical justification of this concept in function space together with the development of a suitable numerical scheme are the subject of future research.

Appendix A. Convergence result for lower level problem. Here we establish the strong convergence result for the state of the lower level minimization problem with associated first order conditions (2.11). With some simplifications a similar proof technique yields the analogous result for $(2.1)$ and (2.6).

TheOrem A.1. Let $y(u) \in H_{0}^{1}(\Omega)$ be the solution of

$$
a(y, v ; u)-\left(\max _{\epsilon}(0, \bar{\lambda}-\gamma y), v\right)=(f(u), v) \quad \forall v \in H_{0}^{1}(\Omega),
$$

where $\bar{\lambda} \in L^{2}(\Omega)$ with $\bar{\lambda} \geq 0$. Further let $\left\{u_{k}\right\}$ be a sequence in $U_{a d}$ which converges weakly in $H^{1}(\Omega)$ to $\bar{u}$. Then $\left\{y\left(u_{k}\right)\right\}$ converges strongly in $H_{0}^{1}(\Omega)$ to $y(\bar{u})$.

\begin{tabular}{|c|c|c|c|c|}
\hline mesh size $h_{y}$ & $1 / 11$ & $1 / 22$ & $1 / 44$ & $1 / 88$ \\
\hline \#it. & 19 & 4 & 3 & 3 \\
\hline
\end{tabular}

TABLE 4.1

Example 2. Iteration numbers for the nested iteration concept. 
Proof. Since $y_{k}:=y\left(u_{k}\right)$ solves (A.1) for every $k$, we have

$$
\left\|y_{k}\right\|_{W_{0}^{1, r}(\Omega)} \leq \beta\left(\left\|f\left(u_{k}\right)\right\|_{W^{-1, r}(\Omega)}+\|\bar{\lambda}\|_{W^{-1, r}(\Omega)}\right)
$$

for some $r>2$ and some positive constant $\beta>0$. As $\left\{u_{k}\right\} \subset U_{\text {ad }}$, we have $\left\|y_{k}\right\|_{W_{0}^{1, r}(\Omega)} \leq \beta$ uniformly in $k$. In particular, there exist $\bar{y} \in H_{0}^{1}(\Omega)$ and a subsequence which we again denote by $\left\{u_{k}\right\}$ such that $y\left(u_{k}\right) \rightarrow \bar{y}$ in $H_{0}^{1}(\Omega)$. For $v \in H_{0}^{1}(\Omega)$ we then have

$$
\begin{aligned}
\mid a\left(y_{k}, v ; u_{k}\right) & -a(\bar{y}, v ; \bar{u})|\leq| a\left(y_{k}, v ; u_{k}\right)-a\left(y_{k}, v ; \bar{u}\right)|+| a\left(y_{k}, v ; \bar{u}\right)-a(\bar{y}, v ; \bar{u}) \mid \\
& \leq\left\|c\left(u_{k}\right)-c(\bar{u})\right\|_{L^{s}(\Omega)}\left\|\nabla y_{k}\right\|_{L^{r}(\Omega)}|| \nabla v \|_{L^{2}(\Omega)}+\left|\int_{\Omega} c(\bar{u}) \nabla\left(y_{k}-\bar{y}\right) \nabla v d x\right| \rightarrow 0 \text { as } k \rightarrow \infty
\end{aligned}
$$

with $s=2 r /(r-2)$. Note that

$$
\left(\max _{\epsilon}\left(0, \bar{\lambda}-\gamma y_{k}\right)-\max _{\epsilon}(0, \bar{\lambda}-\gamma \bar{y}), y_{k}-\bar{y}\right) \leq 0 \quad \forall k .
$$

From this, we infer

$$
\begin{aligned}
0= & a\left(y_{k}, \bar{y}-y_{k} ; u_{k}\right)-a\left(\bar{y}, \bar{y}-y_{k} ; \bar{u}\right)-\left(\max _{\epsilon}\left(0, \bar{\lambda}-\gamma y_{k}\right)-\max _{\epsilon}(0, \bar{\lambda}-\gamma \bar{y}), \bar{y}-y_{k}\right) \\
& -\left(f\left(u_{k}\right)-f(\bar{u}), \bar{y}-y_{k}\right) \\
\leq & a\left(y_{k}-\bar{y}, \bar{y}-y_{k} ; u_{k}\right)+\mid\left(a\left(\bar{y}, \bar{y}-y_{k} ; u_{k}\right)-a\left(\bar{y}, \bar{y}-y_{k} ; \bar{u}\right) \mid\right. \\
& +\left\|f\left(u_{k}\right)-f(\bar{u})\right\|_{L^{2}(\Omega)}\left\|y_{k}-\bar{y}\right\|_{L^{2}(\Omega)}
\end{aligned}
$$

and hence

$$
\left\|y_{k}-\bar{y}\right\|_{H_{0}^{1}(\Omega)}^{2} \leq \beta\left(\mid\left(a\left(\bar{y}, \bar{y}-y_{k} ; u_{k}\right)-a\left(\bar{y}, \bar{y}-y_{k} ; \bar{u}\right) \mid+\left\|y_{k}-\bar{y}\right\|_{L^{2}(\Omega)}\right) .\right.
$$

The right hand side above tends to zero as $k \rightarrow \infty$, which establishes the strong $H_{0}^{1}$-convergence of $\left\{y_{k}\right\}$ to $\bar{y}$. The uniqueness of the solution of (A.1) now implies $\bar{y}=y(\bar{u})$.

Appendix B. Complete continuity of the regularized adjoint state. For the subsequent result we define the control-to-state mapping $y_{\gamma, \epsilon}(\cdot)$ which assigns to $u \in H^{1}(\Omega)$ the unique solution $y_{\gamma, \epsilon}(u)$ of the regularized equation

$$
a(y, v ; u)-\left(\max _{\epsilon}(0, \bar{\lambda}-\gamma y), v\right)-(f(u), v)=0 \quad \forall v \in H_{0}^{1}(\Omega) .
$$

Note that the results of Appendix A yield that $y_{\gamma, \epsilon}(\cdot)$ is completely continuous from $H^{1}(\Omega)$ to $H_{0}^{1}(\Omega)$.

ThEOREM B.1. Let $p_{\gamma, \epsilon}(u) \in H_{0}^{1}(\Omega)$ be the solution of

$$
a(p, v ; u)+\left(\gamma \max _{\epsilon}^{\prime}\left(0, \bar{\lambda}-\gamma y_{\gamma, \epsilon}(u)\right) p, v\right)=(f(u), v) \quad \forall v \in H_{0}^{1}(\Omega),
$$

where $\bar{\lambda} \in L^{2}(\Omega)$ with $\bar{\lambda} \geq 0$. Further let $\left\{u_{k}\right\}$ be a sequence in $U_{a d}$ which converges weakly in $H^{1}(\Omega)$ to $\bar{u}$. Then $\left\{p_{\gamma, \epsilon}\left(u_{k}\right)\right\}$ converges strongly in $H_{0}^{1}(\Omega)$ to $p_{\gamma, \epsilon}(\bar{u})$ as $k \rightarrow \infty$.

Proof. Similar to the proof of Theorem A.1 we obtain the uniform boundedness of $\left\{p_{k}\right\}$ in $W_{0}^{1, r}(\Omega)$ for some $r>2$, with $p_{k}:=p_{\gamma, \epsilon}\left(u_{k}\right)$. Hence, there exists $\bar{p} \in H_{0}^{1}(\Omega)$ and a subsequence again denoted by $\{k\}$ such that $p_{k} \rightarrow \bar{p}$ in $H_{0}^{1}(\Omega)$. This implies

$$
\lim _{k \rightarrow \infty}\left|a\left(p_{k}, v ; u_{k}\right)-a(\bar{p}, v ; \bar{u})\right|=0 .
$$


Next we study

$$
d_{k}(\Omega):=\left\|\left(\max _{\epsilon}^{\prime}(0, \bar{\lambda}-\gamma \bar{y})-\max _{\epsilon}^{\prime}\left(0, \bar{\lambda}-\gamma y_{k}\right)\right) p_{k}\right\|_{L^{2}(\Omega)}^{2}
$$

where we use $y_{k}=y\left(u_{k}\right)$. For this purpose we partition $\Omega$ into several subdomains such that $\Omega=\bigcup_{i=1}^{7} T_{k}^{i}$ with

$$
\begin{array}{ll}
T_{k}^{1}=\left\{\bar{\lambda}-\gamma \bar{y}<-(\epsilon / 2) \wedge \bar{\lambda}-\gamma y_{k}<-(\epsilon / 2)\right\}, & T_{k}^{4}=\left\{|\bar{\lambda}-\gamma \bar{y}| \leq(\epsilon / 2) \wedge\left|\bar{\lambda}-\gamma y_{k}\right| \leq(\epsilon / 2)\right\}, \\
T_{k}^{2}=\left\{\bar{\lambda}-\gamma \bar{y}<-(\epsilon / 2) \wedge\left|\bar{\lambda}-\gamma y_{k}\right| \leq(\epsilon / 2)\right\}, & T_{k}^{5}=\left\{|\bar{\lambda}-\gamma \bar{y}| \leq(\epsilon / 2) \wedge \bar{\lambda}-\gamma y_{k}>(\epsilon / 2)\right\}, \\
T_{k}^{3}=\left\{\bar{\lambda}-\gamma \bar{y}<-(\epsilon / 2) \wedge \bar{\lambda}-\gamma y_{k}>(\epsilon / 2)\right\}, & T_{k}^{6}=\left\{\bar{\lambda}-\gamma \bar{y}>(\epsilon / 2) \wedge \bar{\lambda}-\gamma y_{k}>(\epsilon / 2)\right\}
\end{array}
$$

and $T_{k}^{7}=\Omega \backslash \bigcup_{i=1}^{6} T_{k}^{i}$. On $T_{k}^{7}$ one readily finds

$$
d_{k}\left(T_{k}^{7}\right) \leq \frac{\gamma^{2}}{\epsilon^{2}}\left\|p_{k}\right\|_{L^{2}\left(T_{k}^{7}\right)}^{2}\left\|y_{k}-\bar{y}\right\|_{L^{2}\left(T_{k}^{7}\right)}^{2} \rightarrow 0 \text { for } k \rightarrow \infty .
$$

Here we used Appendix A. We further have that $d_{k}\left(T_{k}^{1}\right)=d_{k}\left(T_{k}^{6}\right)=0$. For $T_{k}^{2}$ we get

$$
d_{k}\left(T_{k}^{2}\right)=\int_{T_{k}^{2}}\left|(1 / \epsilon)\left(\bar{\lambda}-\gamma y_{k}+(\epsilon / 2)\right) p_{k}\right|^{2} d x \leq\left|T_{k}^{2}\right|^{(s-2) / s}\left\|p_{k}\right\|_{L^{s}(\Omega)}^{2}
$$

for some $s>2$. Next we show that $\left|T_{k}^{2}\right| \rightarrow 0$ for $k \rightarrow \infty$. For this purpose define

$$
T_{k}^{2}(\delta):=\left\{\bar{\lambda}-\gamma \bar{y} \leq-(\epsilon / 2)-\delta \wedge\left|\bar{\lambda}-\gamma y_{k}\right| \leq(\epsilon / 2)\right\}
$$

for $\delta>0$. Note that $T_{k}^{2}\left(\delta_{1}\right) \subset T_{k}^{2}\left(\delta_{2}\right) \subset T_{k}^{2}$ for $\delta_{1}>\delta_{2}>0$ and $0>-\delta \geq \gamma\left(y_{k}-\bar{y}\right)$ on $T_{k}^{2}(\delta)$. Then

$$
\left|T_{k}^{2}(\delta)\right| \leq(\gamma / \delta)\left\|y_{k}-\bar{y}\right\|_{L^{1}(\Omega)} \rightarrow 0 \quad \text { for } k \rightarrow \infty
$$

where $\bar{y}=y(\bar{u})$. Since $\delta$ was arbitrary we conclude $\left|T_{k}^{2}\right| \rightarrow 0$ for $k \rightarrow \infty$. Similarly one argues that $d_{k}\left(T_{k}^{3}\right) \rightarrow 0$ for $k \rightarrow \infty$ since

$$
d_{k}\left(T_{k}^{3}\right) \leq\left|T_{k}^{3}\right|^{(s-2) / s}\left\|p_{k}\right\|_{L^{s}(\Omega)}^{2}
$$

for some $s>2$, and

$$
\left|T_{k}^{3}\right| \leq 2 \gamma \epsilon^{-1}\left\|y_{k}-\bar{y}\right\|_{L^{1}(\Omega)}
$$

On $T_{k}^{4}$ we have

$$
d_{k}\left(T_{k}^{4}\right) \leq \beta\left(\gamma^{2} / \epsilon^{2}\right)\left\|\bar{y}-y_{k}\right\|_{L^{4}(\Omega)}^{2} \rightarrow 0 \quad \text { for } k \rightarrow \infty
$$

Here we used Appendix A, again. Finally, for $T_{k}^{5}$ we obtain

$$
d_{k}\left(T_{k}^{5}\right) \leq\left|T_{k}^{5}\right|^{(s-2) / s}\left\|p_{k}\right\|_{L^{s}(\Omega)}^{2}
$$

for some $s>2$. Now one shows similarly to $T_{k}^{2}$ that $\left|T_{k}^{5}\right| \rightarrow 0$ for $k \rightarrow \infty$. Summarizing, we proved that

$$
d_{k}(\Omega) \rightarrow 0 \quad \text { for } k \rightarrow \infty
$$


Hence, the limit point $(\bar{y}, \bar{p}, \bar{u})$ satisfies

$$
a(\bar{p}, v ; \bar{u})-\left(\gamma \max _{\epsilon}^{\prime}(0, \bar{\lambda}-\gamma \bar{y}) \bar{p}, v\right)-(f(\bar{u}), v)=0 \quad \forall v \in H_{0}^{1}(\Omega) .
$$

From this we conclude

$$
\begin{aligned}
0= & a\left(p_{k}, \bar{p}-p_{k} ; u_{k}\right)+\gamma\left(\max _{\epsilon}^{\prime}\left(0, \bar{\lambda}-\gamma y_{k}\right) p_{k}, \bar{p}-p_{k}\right)+\left(y_{k}-y_{m}, \bar{p}-p_{k}\right) \\
& -a\left(\bar{p}, \bar{p}-p_{k} ; \bar{u}\right)-\gamma\left(\max _{\epsilon}^{\prime}(0, \bar{\lambda}-\gamma \bar{y}) \bar{p}, \bar{p}-p_{k}\right)-\left(\bar{y}-y_{m}, \bar{p}-p_{k}\right) \\
\leq & a\left(p_{k}-\bar{p}, \bar{p}-p_{k} ; u_{k}\right)+\left|a\left(\bar{p}, \bar{p}-p_{k} ; \bar{u}\right)-a\left(\bar{p}, \bar{p}-p_{k} ; u_{k}\right)\right| \\
& +\gamma\left\|\left(\max _{\epsilon}^{\prime}(0, \bar{\lambda}-\gamma \bar{y})-\max _{\epsilon}^{\prime}\left(0, \bar{\lambda}-\gamma y_{k}\right)\right) p_{k}\right\|_{L^{2}(\Omega)}\left\|p_{k}-\bar{p}\right\|_{L^{2}(\Omega)}+\left\|y_{k}-\bar{y}\right\|_{L^{2}(\Omega)}\left\|p_{k}-\bar{p}\right\|_{L^{2}(\Omega)}
\end{aligned}
$$

and further infer

$$
\epsilon_{c}\left\|p_{k}-\bar{p}\right\|_{H_{0}^{1}(\Omega)} \leq\left\|c\left(u_{k}\right)-c(\bar{u})\right\|_{L^{t}(\Omega)}\|\nabla \bar{p}\|_{L^{r}(\Omega)}+\gamma \sqrt{d_{k}(\Omega)}+\left\|y_{k}-\bar{y}\right\|_{L^{2}(\Omega)} \rightarrow 0 \quad \text { for } k \rightarrow \infty
$$

with $1 / t+1 / r=1 / 2$ and some $r>2$. The uniqueness of the solution of the regularized adjoint equation now proves the result.

Appendix C. Interior point formulation. In what follows all quantities are discrete. For the ease of notation we neglect the subscript $h$. The interior point formulation (3.7)-(3.9) results from utilizing a barrier approach for the constraints for $u$. The barrier problem corresponding to the discrete version of $(\mathrm{P})$ is given by

$$
\begin{aligned}
& \operatorname{minimize} J(y, u)+\kappa\left(\sum_{i=1}^{n_{h}^{u}} \ln \left(\epsilon_{u}-u_{i}\right)+\sum_{i=1}^{n_{h}^{u}} \ln \left(u_{i}-\epsilon_{l}\right)\right) \text { over }(y, u, \lambda) \in \mathbb{R}^{n_{h}^{y}} \times \mathbb{R}^{n_{h}^{u}} \times \mathbb{R}^{n_{h}^{y}} \\
& \text { subject to } A(u) y-\lambda=f(u), \\
& \qquad \lambda \geq 0, \quad y \geq 0, \quad y \star \lambda=0 .
\end{aligned}
$$

Optimality with respect to $u$ and setting

$$
q_{u, i}:=\kappa\left(\epsilon_{u}-u_{i}\right)^{-1} \quad \text { and } \quad q_{l, i}:=\kappa\left(u_{i}-\epsilon_{l}\right)^{-1}
$$

where the right hand sides result from differentiating the barrier (i.e., the ln-) terms with respect to $u_{i}$, yield $(3.7)-(3.9)$.

\section{REFERENCES}

[1] Y. Achdou. An inverse problem for parabolic variational inequality arising in volatility calibration with American options. SIAM J. Control Optim., 43(5):1583-1615, 2005.

[2] Y. Achdou and O. Pironneau. Computational methods for option pricing, volume 30 of Frontiers in Applied Mathematics. Society for Industrial and Applied Mathematics (SIAM), Philadelphia, PA, 2005.

[3] V. Barbu. Optimal control of variational inequalities, volume 100 of Research Notes in Mathematics. Pitman (Advanced Publishing Program), Boston, MA, 1984.

[4] B. Bayada and M. El Aalaoui Talibi. Control by the coefficients in a variational inequality: the inverse elastohydrodynamic lubrication problem. Report de recherche 173, I.N.S.A. de Lyon, 1994.

[5] A. Bensoussan, J.-L. Lions, and G. Papanicolaou. Asymptotic Analysis of Periodic Structures. North-Holland, Amsterdam, 1978.

[6] G. Cimatti. On a problem of the theory of lubrication governed by a variational inequality. Appl. Math. Optim., $3: 227-242,1997$. 
[7] R. Glowinski. Numerical methods for nonlinear variational problems. Springer Series in Computational Physics. Springer-Verlag, New York, 1984.

[8] J. Guo. A variational inequality associated with a lubrication problem. IMA Preprint Series 530, Institute of Mathematics and its Applications, Minneapolis, Minnesota, USA, 1989.

[9] A. Hasanov. An inverse problem for an elastoplastic medium. SIAM J. Appl. Math., 55(6):1736-1752, 1995.

[10] M. Hintermüller. Inverse coefficient problems for variational inequalities: optimality conditions and numerical realization. M2AN Math. Model. Numer. Anal., 35(1):129-152, 2001.

[11] M. Hintermüller, K. Ito, and K. Kunisch. The primal-dual active set strategy as a semismooth Newton method. SIAM J. Optim., 13(3):865-888, 2003.

[12] M. Hintermüller and K. Kunisch. Path-following methods for a class of constrained minimization problems in function space. SIAM J. Optim., 17(1):159-187 (electronic), 2006.

[13] R. H. W. Hoppe and R. Kornhuber. Adaptive multilevel methods for obstacle problems. SIAM J. Numer. Anal., 31(2):301-323, 1994.

[14] B. Hu. A quasi-variational inequality arising in elastohydrodynamics. SIAM Journal on Math. Anal., 21:18$36,1990$.

[15] N. Kikuchi and J. T. Oden. Contact problems in elasticity: a study of variational inequalities and finite element methods, volume 8 of SIAM Studies in Applied Mathematics. Society for Industrial and Applied Mathematics (SIAM), Philadelphia, PA, 1988.

[16] D. Kinderlehrer and G. Stampacchia. An Introduction to Variational Inequalities and their Applications, volume 31 of Classics in Applied Mathematics. Society for Industrial and Applied Mathematics (SIAM), Philadelphia, PA, 2000. Reprint of the 1980 original.

[17] R. Kornhuber. Adaptive monotone multigrid methods for nonlinear variational problems. Advances in Numerical Mathematics. B. G. Teubner, Stuttgart, 1997.

[18] Z.-Q. Luo, J.-S. Pang, and D. Ralph. Mathematical Programs with Equilibrium Constraints. Cambridge University Press, Cambridge, United Kingdom, 1996.

[19] J. Outrata, M. Kočvara, and J. Zowe. Nonsmooth approach to optimization problems with equilibrium constraints, volume 28 of Nonconvex Optimization and its Applications. Kluwer Academic Publishers, Dordrecht, 1998. Theory, applications and numerical results.

[20] J. Outrata and J. Zowe. A numerical approach to optimization problems with variational inequality constraints. Math. Prog., 68:105-130, 1995.

[21] J.-S. Pang, S. Han, and N. Rangaraj. Minimization of locally Lipschitz functions. SIAM J. Optimization, 1:57-92, 1991.

[22] J.-S. Pang and L. Qi. A globally convergent Newton method for convex SC ${ }^{1}$ minimization problems. J. Optimization Theory Appls., 85:633-648, 1995.

[23] J.-F. Rodriguez. Obstacle Problems in Mathematical Physics, volume 134 of Mathematics Studies. NorthHolland, Amsterdam, 1987.

[24] H. Scheel and S. Scholtes. Mathematical programs with complementarity constraints: stationarity, optimality, and sensitivity. Math. Oper. Res., 25(1):1-22, 2000.

[25] S. Scholtes. Convergence properties of a regularization scheme for mathematical programs with complementarity constraints. SIAM J. Optim., 11(4):918-936 (electronic), 2001.

[26] S. J. Wright. Primal-dual Interior-Point Methods. Society for Industrial and Applied Mathematics (SIAM), Philadelphia, PA, 1997.

[27] J. Zowe and S. Kurcyusz. Regularity and stability for the mathematical programming problem in Banach spaces. Appl. Math. Optim., 5(1):49-62, 1979. 